\title{
Efficient Detection and Signal Parameter Estimation With Applications to High Dynamic GPS Receivers
}

R. Kumar

\author{
(BASA-CE-1848(1) EFFICIEAI LEIECIICN AND \\ $\triangle 89-2 C \equiv \in 2$ \\ SIGAAL EAEAEETEE FSTIEATICB LIE \\ DEFLICAIICAS TC EIGE CINAAIC CES EECEIVEES \\ (Jet Erofulsice Lat.) $40 \mathrm{~F}$ CSCL 17B \\ Unclas \\ $019 \varepsilon E 56$
}

December 15, 1988

\author{
Prepared for \\ U.S. Air Force Systems Command \\ Armament Division \\ Through an agreement with \\ National Aeronautics and \\ Space Administration \\ by \\ Jet Propulsion Laboratory \\ California Institute of Technology \\ Pasadena, California
}




\title{
Efficient Detection and Signal Parameter Estimation With Applications to High Dynamic GPS Receivers
}

\author{
R. Kumar
}

December 15, 1988

Prepared for

U.S. Air Force Systems Command

Armament Division

Through an agreement with

National Aeronautics and

Space Administration

by

Jet Propulsion Laboratory California Institute of Technology

Pasadena, California 
The research described in this publication was carried out by the Jet Propulsion Laboratory, California Institute of Technology, under contract with the National Aeronautics and Space Administration, in part sponsored by the United States Air Force Systems Command, through an agreement with the National Aeronautics and Space Administration, under Task Order RE-182, Amendment 452.

Reference herein to any specific commercial product, process, or service by trade name, trademark, manufacturer, or otherwise, does not constitute or imply its endorsement by the United States Government or the Jet Propulsion Laboratory, California Institute of Technology. 


\begin{abstract}
This publication presents a novel technique for simultaneously detecting data and estimating the parameters of a received carrier signal phase modulated by unknown data and experiencing very high Doppler, Doppler rate, etc. Such a situation arises, for example, in the case of Global Positioning Systems (GPS) where the signal parameters are directly related to the position, velocity and acceleration of the GPS receiver.

The proposed scheme is based upon first estimating the received signal local (data dependent) parameters over two consecutive bit periods, followed by the detection of a possible jump in these parameters. The presence of the detected jump signifies a data transition which is then removed from the received signal. This effectively demodulated signal is then processed to provide the estimates of global (data independent) parameters of the signal related to the position, velocity, etc. of the receiver.

One of the key features of the proposed algorithm is the introduction of two different schemes which can provide an improvement of up to $3 \mathrm{~dB}$ over the conventional implementation of Kalman filter as applied to phase and frequency estimation, under low to medium signal-to-noise ratio conditions. One scheme is based upon reprocessing (cycling) the measurements over an optimally selected interval while the alternative scheme proposes an adaptive Hilbert transform technique. The overall complexity of the proposed algorithm is about three times the complexity of a single third order Kalman filter.
\end{abstract}




\section{CONTENTS}

1. INTRODUCTION

2. THE SIGNAL MODEL $\quad 4$

Optimum Solutions $\quad 4$

3. SIMULTANEOUS ESTIMATION AND DETECTION SCHEME $\quad 7$

4. ESTIMATION ALGORITHM (RECURSION IN SAMPLES) 11

Improved Kalman Filter 11

Modified Phase Locked Loop with up to $3 \mathrm{~dB}$ Improvement 13

5. SIMULATIONS 15

$\begin{array}{llr}\text { 6. CONCLUSIONS } & 18\end{array}$

$\begin{array}{lr}\text { REFERENCES } & 19\end{array}$

$\begin{array}{ll}\text { APPENDIX } & 21\end{array}$

Figures

1. Illustrative Received Signal Waveform 23

2. Schematic Diagram of the Proposed Estimation/
Detection Scheme

3. Modified Phase-Locked Loop with up to $3 \mathrm{~dB}$ Improvement 25

4a. Errors in Estimates of Frequency at Bit Intervals
(Acceleration $50 \mathrm{~g}$ )

4b. Errors in Estimates of Frequency Derivative at

4c. Carrier Phase Estimation Error at the Start of

Bit Periods (Acceleration 50g) 28

5a. Errors in Estimates of Frequency at Bit Intervals with
Data Detection (Acceleration 50g)

5b. Errors in Estimates of Frequency Derivative at $50 \mathrm{~g}$ )

6a. Errors in Estimates of Frequency at Bit Intervals

6b. Errors in Estimates of Frequency Derivative

(Acceleration 50g) 32

7a. Errors in Estimates of Frequency at Bit Intervals

7b. Errors in Estimates of Frequency Derivative at
Bit Intervals (Acceleration 100g) 


\section{INTRODUCTION}

The problem of estimating the parameters of a received quasi-sinusoidal signal in the presence of noise has received considerable attention in the literature [1-12]. However, for the case when the received carrier is modulated by unknown data and simultaneously experiences considerably high Doppler and Doppler rate, the research reported in the published literature is somewhat limited. This situation arises, for example, in the case of Global Positioning Systems (GPS) receivers exhibiting very high dynamics [7, 12].

In a previous publication [7], an estimator structure based on the maximum likelihood estimation (MLE) of code delay and Doppler frequency over a single data bit period has been proposed and analyzed for the GPS application. The "pseudo" estimates over different bit intervals are combined by a Kalman filter to provide tracking of Doppler frequency. By limiting the primary (MLE) estimation period to less than one data bit period, the problem of detecting the data bits is bypassed. However, perhaps due to such a limitation and also due to the high frequency rate involved (not explicitly estimated by the MLE), a threshold of about $30 \mathrm{~dB}-\mathrm{Hz}$ in terms of the received carrier power to noise power spectral density ratio $\left(P / N_{0}\right)$ was obtained. Due to the lack of knowledge of data bit, phase estimation is not feasible in this scheme.

In terms of GPS applications, the problem of data modulation can be overcome by establishing a parallel (non-dynamic) link between the GPS satellites and a control ground receiver which also simultaneously receives the frequency translated version of the GPS receiver signals [13]. The data demodulation and estimation is then performed at the ground receiver. Once the data modulation is removed from the GPS receiver signal, then the problem reduces to simply estimating the phase, frequency, etc. of an unmodulated r-f carrier. This latter problem of course, has been extensively studied in the literature. See for example [9] for a very efficient method for simultaneous estimation of frequency and its derivative, and [12] for some similar techniques, including a third and fourth order Kalman filter, as applied to the GPS receiver problem.

In this publication, we address the original (and more difficult) problem of estimating the signal parameters from a data modulated sinusoidal carrier and propose a novel simultaneous estimation-detection scheme whose performance is very close to the estimation schemes proposed for the case of the unmodulated carrier. In fact, for the case of high dynamics under consideration, there is virtually no loss in terms of the required $\left(P / N_{0}\right)$ due to data modulation. Apart from the fact that in GPS applications this does away with 
the necessity of having a parallel direct satellite to ground receiver communication link, the proposed scheme is very important in many other similar situations including (modulated) signal acquisition from NASA deep space probes undergoing high dynamics, where such parallel links may not be feasible.

In the literature $[14,15]$ there are several techniques of data detection. If the signal waveform is known precisely (and does not change from bit to bit) then data can be detected coherently by using matched filters or correlation receivers, irrespective of the actual waveform. If the waveform (carrier) has a constant known frequency, then either a coherent or differentially coherent detection may be employed depending upon whether or not the phase of the carrier is known. In a decision-directed version of these techniques, the carrier phase and/or frequency are estimated by a phase-locked loop technique and the data detector becomes part of the loop. It is clear that these techniques may not be feasible under low signal-to-noise ratio and the high dynamics considered here, in that the frequency may not be even nearly constant over the detection period and under such low loop SNR conditions it may not be possible to acquire the lock with data modulation present.

In an alternative solution as in the Costas loop [15], the data detection problem is bypassed by a multiplicative technique. However, such a loop also suffers in terms of loss of the SNR due to the multiplicative noise term which can be excessive for high loop filter bandwidths required and low received SNR.

It is thus apparent that schemes which incorporate data detection in a loop which in turn is made dependent upon the acquisition and tracking of the loop may not be desirable under such high dynamics conditions since the loop may not acquire to start with (and may lose lock during tracking). Therefore, in this publication we propose and investigate techniques where data can be detected even under open loop condition. The fact that this information can subsequently be used to obtain an overall closed-loop configuration is only of secondary importance.

The proposed scheme is based upon estimation of the received signal "local" (data dependent) parameters over two consecutive bit periods, followed by a detection of a possible jump in these parameters. The presence of the detected jump signifies a data bit transition which is removed from the received signal. This effectively demodulated signal is then processed to provide the estimates of "global" (data independent) parameters of the signal which also provide a prediction for the "local" parameters over subsequent bit 
periods.

The estimation algorithm for both the "local" and "global" parameters estimation is an improved version of the Kalman filter. For "local" parameter estimation it is necessary to use an algorithm capable of estimating both the phase and frequency (data is phase modulated). However, for the "global" estimation algorithm it is not necessary to estimate the phase and thus the Kalman filter may be substituted by the frequency estimation algorithm of [9], resulting in a marginal reduction of the required $P / N_{0}$.

One of the key features of the proposed algorithm is the introduction of two different schemes which can provide an improvement of up to $3 \mathrm{~dB}$ over the Kalman filter (and phase or frequency locked loops) as applied to phase and frequency estimation under low signal-to-noise ratio (SNR) conditions. Both of these schemes are based upon the recognition of two available error signals with nearly independent noise. In conventional implementations, one of these error signals is simply ignored. Here we propose that by reprocessing the observations over an optimally selected period, one can exploit the other error signal as well. It may be emphasized that the improvement here is not due to better linearization as in iterated Kalman filter (where the improvement increases with the iteration interval) but rather due to the fact that if the reprocessing interval is optimal, the two sets of measurements are nearly independent, resulting in nearly 3 dB improvement of performance under low SNR. In an alternative scheme, relevant to classical phase-locked loop structures, we propose an adaptive Hilbert transform technique resulting in similar improvements.

In section 2 we present the signal model with the optimal solution, followed by the proposed simultaneous estimation and detection scheme in section 3. Section 4 presents the estimation algorithm in some details with the two proposed modifications. In section 5 we give simulation results where the algorithm is applied to the GPS receiver parameter estimation problem. The last section of the publication contains some concluding remarks. 


\section{THE SIGNAL MODEL}

Consider the problem of simultaneously estimating the high dynamic phase process $\Theta(t)$ and detecting the data modulation from the measurements $y(t), z(t)$ in (1) made in the presence of zero mean additive white Gaussian noise $n_{i}(t), n_{q}(t)$.

$$
\begin{aligned}
& y(t)=A \operatorname{Sin}(\Theta(t)+\pi D(t))+v_{i}(t) \\
& z(t)=A \operatorname{Cos}(\Theta(t)+\pi D(t))+v_{q}(t) \quad ; \quad 0 \leq t \leq T_{0}
\end{aligned}
$$

In (1) $D(t)$ is a binary digital waveform taking possible values 0 or 1 . Assume that over a sufficiently small estimation period,

$$
\Theta(t)=\theta_{0}+\omega_{0} t+\gamma_{0} t^{2}+\delta_{0} t^{3}
$$

for some unknown parameter vector $\psi_{0}=\left[\begin{array}{llll}\theta_{0} & \omega_{0} & \gamma_{0} & \delta_{0}\end{array}\right]^{\prime}$. Thus $\{y(t), z(t)\}$ represent the in-phase and quadrature components of a received signal $s(t)$ and $\omega_{0}$ is the offset between the frequencies $f_{c}$ of the carrier reference signal $r(t)$ and the received signal representing the Doppler, while the Doppler rate is equal to $2 \gamma_{0}$ etc. In case of positioning systems, the phase derivative $\dot{\Theta}(t)$ is related to the physical velocity trajectory $v_{d}(t)$ of the receiver as

$$
\dot{\Theta}(t) \cong 2 \pi \frac{f_{c}}{c} v_{d}(t)
$$

where $c$ is the velocity of light. Similar normalization of the second and third derivatives of $\dot{\Theta}(t)$ yields the acceleration and jerk (second derivative of velocity) respectively of the physical trajectory.

Sampling the inphase and quadrature signal waveforms $y(t)$ and $z(t)$ at a rate of $1 / T$, one obtains

$$
\begin{aligned}
& y(k)=A \operatorname{Sin}(\Theta(k)+\pi D(k))+v_{i}(k) \\
& z(k)=A \operatorname{Cos}(\Theta(k)+\pi D(k))+v_{q}(k) \quad ; \quad D(k)=0,1
\end{aligned}
$$

The signal $y(t)$ (and similarly $z(t)$ ) may be depicted as in Figure 1 .

\section{Optimum Solutions}

It is desired to obtain the estimate of parameter vector $\psi_{0}$ and detect data bits $d_{1}, \ldots, d_{n}$ on the basis of measurements $Y(M), Z(M) \cong\{y(k), z(k) ; k=1, \ldots, M\}$ where $\mathrm{M}=\mathrm{Nn}, \mathrm{n}$ is the number of received 
digits and $\mathrm{N}$ is the number of samples/bit. The optimum solution can be obtained by maximizing the a-posteriori probability density function of $\psi_{0}, d_{1}, \ldots, d_{n}$ conditioned upon $Y(N), Z(N)$ and denoted by $p\left(\psi_{0}, d_{1}, \ldots, d_{n} / Y(M), Z(M)\right)$. Such a maximization can be achieved by first computing the $2^{n}$ possible conditional probability density functions $p_{i} \triangleq \max _{\psi_{0}} p\left(\psi_{0} \mid Y(N), Z(N) ; \mathcal{S}_{i}\right)$ at their respective optimum point $\hat{\psi}_{i}$. In the expression for $p_{i}, \mathcal{S}_{i}$ denotes the ith possible binary sequence $\mathcal{S}_{i_{1}}, \ldots, \mathcal{S}_{i_{n}}$ for the received digits. This is followed by selecting the maximum out of these $2^{n}$ values. Thus if $p_{m}=\max \left(p_{1}, \ldots, p_{2} n\right)$ for some $m$ between 1 and $2^{n}$, then $\hat{\psi}_{0}^{\text {opt }}=\hat{\psi}_{m}$ and $\hat{d}_{1}, \ldots, \hat{d}_{n}=\mathcal{S}_{m}$.

To evaluate the ith probability $p_{i}$, obtain the set of modified measurements $\bar{Y}(M), \bar{Z}(M)$ defined as

$$
\begin{aligned}
& \bar{y}(k)=\mathcal{S}_{i j} y(k) \\
& \bar{z}(k)=\mathcal{S}_{i j} z(k) \quad ; \quad(j-1) N+1 \leq k \leq j N \quad ; \quad j=1, \ldots, n
\end{aligned}
$$

and then computing $p_{i}=\max _{\psi_{0}} p\left(\psi_{0} \mid \bar{Y}(M), \bar{Z}(m)\right)$.

When there is no a-priori knowledge about $\psi_{0}$, one may instead maximize $p\left(\bar{Y}(M), \bar{Z}(M) \mid \hat{\psi}_{0}\right)$ with respect to $\hat{\psi}_{0}$. In case of Gaussian additive noise considered here, such a maximization is equivalent to minimization of the sum of prediction error squares, i.e., one simply minimizes

$$
\sum_{k=1}^{M}\left\{[\bar{y}(k)-A \operatorname{Sin}(\hat{\Theta}(k))]^{2}+[\bar{z}(k)-A \operatorname{Cos}(\hat{\Theta}(k))]^{2}\right\}
$$

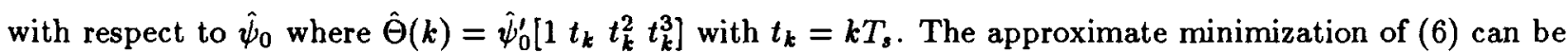
achieved by a recursive algorithm like the Kalman filter.

In this publication we present a computationally simpler suboptimal version of the optimal estimator given above. Such a simplification is achieved by considering the case of $n=1$ first and observing that one could estimate a modified parameter vector $\bar{\psi}_{0} \triangleq \psi_{0}+l d_{1} \pi$ with $l^{\prime} \triangleq\left[\begin{array}{llll}1 & 0 & 0 & 0\end{array}\right]$, directly without an explicit knowledge of the data bit $d_{1}$. The estimate of $\bar{\psi}_{0}$ in turn permits the computation of the total phase $\bar{\Theta}(k)=d_{1} \pi+\Theta(k)$ at the boundary of first bit $\left(t=T_{b}-\right)$. A similar computation with the measurements in the second bit interval permits the computation of total phase $d_{2} \pi+\Theta(k)$ at $t=T_{b}+$. Now it is obvious (see figure 1) that $\Theta(k)$ does not have any discontinuity at $t=T_{b}$ and thus any difference among the above two estimates can result either due to data transition or noise. Thus, if the signal-to-noise ratio is adequate, it is then possible to detect such a data transition with "small" probability of error. In this case the transition 
can be eliminated from the measurements during the second bit period and the estimation of $\bar{\psi}_{0}$ can be updated on the basis of the additional measurements.

Assuming that the first bit is zero (without any restriction on the algorithm) the above decision-directed method effectively removes data modulation and provides at time $\mathbf{k}$, the following modified measurement.

$$
\begin{aligned}
& \bar{y}(k-N) \triangleq A \operatorname{Sin}(\Theta(k-N)+\pi D(k-N)+\pi \hat{D}(k-N)) \pm v_{i}(k-N) \\
& \bar{z}(k-N) \triangleq A \operatorname{Cos}(\Theta(k-N)+\pi D(k-N)+\pi \hat{D}(k-N)) \pm v_{q}(k-N)
\end{aligned}
$$

where $\mathrm{N}$ represents the delay equal to the number of samples in one bit period introduced in the physical realization and $\hat{D}(k)$ is an estimate of $D(k)$. If $\hat{D}(k)=D(k)$ then of course, equation (7) reduces to

$$
\begin{aligned}
& \bar{y}(k)=A \operatorname{Sin}\left(\theta_{0}+\omega_{0} T_{s} k+\gamma_{0} T_{s}^{2} k^{2}+\delta_{0} T_{s}^{3} k^{3}\right)+\bar{v}_{i}(k) \\
& \bar{z}(k)=A \operatorname{Cos}\left(\theta_{0}+\omega_{0} T_{s} k+\gamma_{0} T_{s}^{2} k^{2}+\delta_{0} T_{s}^{3} k^{3}\right)+\bar{v}_{q}(k)
\end{aligned}
$$

From (8) one can easily estimate $\omega_{0}, \gamma_{0}, \delta_{0}$ etc. for example, by an extended Kalman filter. The effect of any decision errors made in the detection of $D(k)$ would be to introduce errors in the parameter estimates. But intuitively, such an effect would be most significant upon $\theta_{0}$, a parameter which is not significant in itself under the present application. 


\section{SIMULTANEOUS ESTIMATION AND DETECTION SCHEME}

In this section, we present a novel combined estimation/detection scheme which simultaneously detects data bits and obtains estimates of signal parameters such as carrier phase, frequency, etc. (related to receiver dynamics) in a sequential manner. The proposed scheme is recursive in both the number of data bits and the observations within any one data bit. The procedure described here effectively involves two mutually coupled estimation processes. In one of these estimation processes, we obtain the estimates of the signal parameters (phase, frequency, etc.) in the vicinity of possible data transitions (symbol boundaries) on the basis of measurements obtained within the current data bit. These estimates, which are dependent upon both the data and the receiver dynamics, and termed "local" parameter estimates, are then used to decide whether or not a data transition has actually occurred. On the basis of this information, data modulation is removed from the received signal and the modified observations are reprocessed to update the "global" parameters (dependent only upon the receiver dynamics and independent of data modulation) by taking into account the additional observations during the current "detected" bit.

A block diagram of the proposed estimation/detection scheme is depicted in Figure 2. It may be noted that a closed-loop configuration involving the feedback correction signal to the reference numerically controlled oscillator (NCO) is required to keep the signal frequency $\dot{\Theta}(t)$ at the phase detector output within the bandwidth of the filter (accumulator) following the phase detector. Such a correction at a rate equal to a submultiple of bit rate consists of simply transferring the estimate of frequency (and possibly that of frequency derivative as well) to the NCO. In the following, we first describe in some detail the overall estimation/detection scheme without specific details of the estimation algorithm itself. In this paper we apply a modified Kalman filter but other appropriate recursive parameter estimation algorithms may be adapted to the proposed framework. The proposed estimation/detection scheme involves the following recursive (in number of data bits) steps.

\section{Recursion in Data Bits}

Step 1: Let $\mathrm{N}$ be the total number of samples in any one bit period (assumed to be an integer) with the first sample appearing at time $t=0^{+}$and the Nth sample occurring at time $T_{b}-T$, where $T_{b}$ denotes the bit period. With a recursive algorithm to be described subsequently, we obtain the estimates of the parameter 
vector $\bar{\psi}_{0} \triangleq\left[\begin{array}{llll}\bar{\theta}_{0} & \omega_{0} & \gamma_{0} & \delta_{0}\end{array}\right]^{T}$ at time $t=0^{+}$on the basis of first $\mathrm{N}$ measurements. Here $\bar{\theta}_{0} \triangleq \theta_{0}+\pi d_{1}$ with $d_{1}$ denoting the first data bit with its value 1 or 0 , i.e. $\bar{\theta}_{0}$ denotes the total phase at $t=0^{+}$.

Step 2: In order to detect bit transitions we need to obtain estimates of "local" parameters at the bit boundaries. Thus to detect the possible transition at the end of first data bit period, it is required to obtain estimates of the parameters at $t=T_{b}-$ and $t=T_{b}+$. Noting that the parameters at $t=T_{b}-$ are related to the corresponding parameters at $t=0^{+}$by the following linear transformation,

$$
\bar{\psi}\left(T_{b}-\right) \triangleq\left[\begin{array}{c}
\bar{\theta}\left(T_{b}-\right) \\
\omega\left(T_{b}-\right) \\
\gamma\left(T_{b}^{-}\right) \\
\delta\left(T_{b}-\right)
\end{array}\right]=\left[\begin{array}{cccc}
1 & T_{b} & T_{b}^{2} & T_{b}^{3} \\
0 & 1 & 2 T_{b} & 3 T_{b}^{2} \\
0 & 0 & 1 & 3 T_{b} \\
0 & 0 & 0 & 1
\end{array}\right]\left[\begin{array}{c}
\bar{\theta}_{0} \\
\omega_{0} \\
\gamma_{0} \\
\delta_{0}
\end{array}\right]
$$

one could predict the estimate of parameter vector $\bar{\psi}\left(T_{b}^{-}\right)$via the same linear transformation, i.e.:

$$
\begin{aligned}
& \hat{\bar{\psi}}_{L}\left(T_{b}-\right)=S\left(T_{b}\right) \hat{\bar{\psi}}_{0} \\
& \hat{\bar{\psi}}_{P}\left(T_{b}-\right)={ }_{2 \pi}^{\bmod }\left(\hat{\bar{\psi}}_{L}\left(T_{b}^{-}\right)+l \frac{\pi}{2}\right)
\end{aligned}
$$

where $S\left(T_{b}\right)$ denotes the matrix appearing in $(9), l^{\prime} \triangleq\left[\begin{array}{llll}1 & 0 & 0 & 0\end{array}\right]$ and modulo $2 \pi$ operation is performed on the first component of the argument.

However, since we are interested in optimum modulo $2 \pi$ estimate of the phase $\bar{\theta}\left(T_{b}-\right)$, a nonlinear function of $\bar{\psi}_{0}$, the modulo $2 \pi$ version of $\hat{\bar{\psi}}_{L}\left(T_{b}-\right)$ appearing in (10) and denoted a $\hat{\bar{\psi}}_{P}\left(T_{b}-\right)$ is not optimal even if $\hat{\bar{\psi}}_{0}$ is optimum. Also in the subsequent kth bit period, the predicted estimate of the phase could differ by $\pi$ due to data modulation if $d_{k} \neq d_{0}$. Thus we simply set $\hat{\bar{\theta}}_{P}\left(T_{b}-\right)$ equal to its predicted value plus $\pi / 2$ or it may simply be set to zero.

An optimum modulo $2 \pi$ estimate of $\bar{\psi}\left(T_{b}-\right)$ is obtained by reprocessing the first $\mathrm{N}$ measurements but with their time and phase reference measured with respect to $t=T_{b}-$ as follows. With the initial estimate given by $\hat{\bar{\psi}}_{P}\left(T_{b}-\right)$, apply the recursive estimation algorithm to the set of following measurements to obtain an optimum filtered estimate of $\bar{\psi}\left(T_{b}-\right)$ denoted $\hat{\bar{\psi}}_{F}\left(T_{b}-\right)$.

$$
\begin{aligned}
& y(j)=A \operatorname{Sin}\left(\Theta_{L, 1}(j)\right)+v_{i}(j) \\
& z(j)=A \operatorname{Cos}\left(\Theta_{L, 1}(j)\right)+v_{q}(j) \quad ; j=-N,-(N-1) \cdots,-1 \\
& \Theta_{L, 1}(j)=\bar{\theta}\left(T_{b}-\right)+j T_{s} \omega\left(T_{b}-\right)+\left(j T_{s}\right)^{2} \gamma\left(T_{b}-\right)+\left(j T_{s}\right)^{3} \delta\left(T_{b}-\right)
\end{aligned}
$$


We note that in (11), $\bar{\theta}\left(T_{b}-\right)$ denotes modulo $2 \pi$ phase at $t=T_{b}-$ and thus $\Theta_{L, 1}(j)$ may differ from $\Theta(N+j)$ by an integer multiple of $2 \pi$. The fact that the error covariance of $\hat{\bar{\psi}}_{F}\left(T_{b}-\right)$ may differ from the error covariance of $\hat{\bar{\psi}}_{P}\left(T_{b}-\right)$ is apparent from the fact that the Cramer Rao error bounds are dependent on the time reference [1].

Step 3: assuming that all the parameters except $\bar{\theta}$ cannot change instantaneously, the predicted estimates of parameters at $t=T_{b}+$ (just after the possible data transition) are given by

$$
\begin{array}{ll}
\hat{\omega}_{P}\left(T_{b}+\right)=\hat{\omega}_{F}\left(T_{b}-\right) & ; \quad \hat{\gamma}_{P}\left(T_{b}+\right)=\hat{\gamma}_{F}\left(T_{b}-\right) \\
\hat{\delta}_{P}\left(T_{b}+\right)=\hat{\delta}_{F}\left(T_{b}-\right) & ; \quad \hat{\bar{\theta}}_{P}\left(T_{b}+\right)=\hat{\bar{\theta}}_{F}\left(T_{b}-\right)+\pi / 2
\end{array}
$$

With the initial estimate given by $\hat{\bar{\psi}}_{P}\left(T_{b}+\right)$ in (12), apply the recursive estimation algorithm to the set of following measurements $\{\bar{y}(k), \bar{z}(k) \quad ; \quad k=1,2, \cdots, N\}$ to obtain an optimum filtered estimate of $\psi\left(T_{b}+\right)$ denoted $\hat{\psi}_{F}\left(T_{b}+\right)$. Under low SNR conditions $\hat{\bar{\theta}}_{P}\left(T_{b}+\right)$ in (12) may be set to zero.

$$
\begin{aligned}
& \bar{y}(j)=y(N+j)=A \operatorname{Sin}\left(\Theta_{L, 2}(j)\right)+v_{i}(j) \\
& \bar{z}(j)=z(N+j)=A \operatorname{Cos}\left(\Theta_{L, 2}(j)\right)+v_{q}(j) \quad ; \quad j=0,1 \cdots, N-1 \\
& \Theta_{L, 2}(j)=\bar{\theta}\left(T_{b}+\right)+j T_{s} \omega\left(T_{b}+\right)+\left(j T_{s}\right)^{2} \gamma\left(T_{b}+\right)+\left(j T_{s}\right)^{3} \delta\left(T_{b}+\right)
\end{aligned}
$$

In equations $(11,13)$ suffix $L$ (Local) on $\Theta$ refers to the fact that the cumulative phase $\Theta$ is measured with reference to time $T_{b}-$ and $T_{b}+$ respectively. For computational simplicity, $\hat{\omega}_{F}\left(T_{b}-\right)$ etc. in (12) may be replaced by the corresponding components of $\hat{\bar{\psi}}_{P}\left(T_{b}-\right)$ of (10) and step 2, 3 may thus be performed concurrently.

Step 4: We note that irrespective of the dynamics involved (excluding the physically impossible case of instantaneous position change), $\bar{\theta}\left(T_{b}+\right)$ can differ from $\bar{\theta}\left(T_{b}-\right)$ only if a data transition occurs at $t=T_{b}$, i.e. $d_{2} \neq d_{1}$. In case of no transition we have that $d_{2}=d_{1}$ and $\bar{\theta}\left(T_{b}+\right)=\bar{\theta}\left(T_{b}-\right)$. Thus we apply the following simple decision rule for data detection.

$$
\begin{aligned}
\hat{d}_{2} & =\hat{d}_{1} \quad \text { if } \quad\left|\bar{\theta}\left(T_{b}+\right)-\bar{\theta}\left(T_{b}-\right)\right|<\pi / 2 \\
& \neq \hat{d}_{1} \quad \text { otherwise }
\end{aligned}
$$

A detection error thus occurs only if the phase estimation error is greater than $\pi / 2$. 
Step 5: In this step, the estimate of the global parameter vector $\psi_{0}$ (parameters at $t=0^{+}$) is updated on the basis of modified measurements $\left\{y_{M}(k), z_{M}(k) ; k=N, \cdots, 2 N-1\right\}$, where $y_{M}(k)=y(k)$ if $\hat{d}_{2}=\hat{d}_{1}$ and $y_{M}(k)=-y(k)$ otherwise, with similar expression for $z_{M}(k)$. Thus if there is no detection error made, one would obtain the same estimate for $\psi_{0}$ as for the case of no data modulation. Any error in data detection simply appears as additional state noise in the algorithm. The significance of this step is that this yields (near optimum) estimation of the absolute phase $\hat{\bar{\Theta}}_{0}\left(0^{+} / 2 T_{b}-\right)$, as against modulo $2 \pi$ phase, and from this absolute phase estimate $\hat{\Theta}_{\mathrm{abs}}\left(t / 2 T_{b}-\right.$ ) (carrier phase in the absence of any data modulation) at any time $t$ can be determined by simple linear transformation $S(t)$ defined in (9). Equivalently using a dynamic model for phase [11], one could directly estimate total phase $\Theta(k)$ at any time instance $k$.

Whereas by necessity, $\hat{\bar{\theta}}\left(T_{b}+\right)$ is based upon only the measurements $\{y(k), z(k) \quad ; \quad N \leq k \leq 2 N-1\}$ (previous measurements influence this only indirectly via $\hat{\omega}\left(T_{b}-\right)$ etc.); the phase estimate $\hat{\Theta}_{\mathrm{abs}}\left(t / 2 T_{b}-\right.$ ) is obtained on the basis of complete set of measurements up to instance $2 N-1$ and refers to the carrier phase in the absence of data modulation (assuming that the first bit is 0 ). This more accurate phase estimate can then be used to obtain a more accurate (coherent) detection of data if a delay on data detection but not on parameter estimation can be introduced.

Step 6: Steps 2-4 are now repeated for detection of subsequent data transitions and step 5 updates the estimate of $\psi_{0}$ on the basis of additional measurements during consecutive bit intervals. Note that in the first step of the algorithm, one would have

$$
\hat{\bar{\psi}}\left(k T_{b}-\right)=S\left(k T_{b}\right) \hat{\bar{\psi}}\left(0^{+} / k T_{b}-\right) \quad ; k=1,2 \ldots
$$

The generalization of other steps of the algorithm is obvious. 


\section{ESTIMATION ALGORITHM (RECURSION IN SAMPLES)}

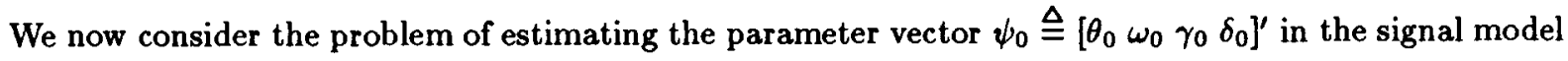
(1-4) when the data modulation has been removed as described above. For this purpose, the signal model is written in the following state space form.

$$
\begin{gathered}
x(k+1)=\Phi x(k)+w(k) \\
Z(k)=h(x(k), k)+v(k) \\
Z(k)=\left[\begin{array}{l}
y(k) \\
z(k)
\end{array}\right] \quad ; \quad v(k)=\left[\begin{array}{l}
v_{i}(k) \\
v_{q}(k)
\end{array}\right] ; \quad h(x(k), k)=\left[\begin{array}{l}
A \operatorname{Sin}(\Theta(k)) \\
A \operatorname{Cos}(\Theta(k))
\end{array}\right] \\
\Theta(k)=J^{\prime}(k) x(k) \quad, \quad J^{\prime}(k)=\left[\begin{array}{llll}
1 & t_{k} & t_{k}^{2} & t_{k}^{3}
\end{array}\right] \\
x^{\prime}(k)=\left[\begin{array}{llll}
\theta_{0} & \omega_{0} & \gamma_{0} & \delta_{0}
\end{array}\right] ; \quad \Phi=I
\end{gathered}
$$

where I denotes the identity matrix and $\{v(k)\},\{w(k)\}$ are assumed to be zero mean white Gaussian noise processes with covariances given by

$$
E\left[\omega(k) \omega^{\prime}(k)\right]=Q \quad, \quad E\left[v(k) v^{\prime}(k)\right]=R=\left[\begin{array}{cc}
r & 0 \\
0 & r
\end{array}\right]
$$

Model (15) is similar to those of $[11,16]$ except that here we treat the model state as the unknown parameter vector $\psi_{0}$ rather than the time-varying phase and frequency process. The two models are completely equivalent if there is no data modulation or in case of modulation, no detection errors are made. As shown in the appendix, the extended Kalman filter equations for the above model are given by

$$
\hat{x}(k+1 / k+1)=\Phi \hat{x}(k / k)+K(k+1) \in(k+1)
$$

where the gain $K(k+1)$ does not depend upon the parameter estimates. The updates for the gain $K(k+1)$ and the prediction error $\in(k+1)$ are given by equations (A10, A12) of the appendix.

Improved Kalman Filter:

In the following, we propose a novel (and simple) modification to the Kalman filter, which results in about $3 \mathrm{~dB}$ improvement in terms of signal-to-noise ratio. It is observed that the prediction error $\in(k)$ in (17) may be written as 


$$
\in(k)=A \operatorname{Sin}(\tilde{\Theta}(k))+n_{I}(k)
$$

where

$$
\tilde{\Theta}(k)=\Theta(k)-\hat{\Theta}(k) \quad ; \quad n_{I}(k)=v_{i}(k) \operatorname{Cos}(\hat{\Theta}(k))-v_{q}(k) \operatorname{Sin}(\hat{\Theta}(k))
$$

Now, we define a related error term $\eta(k)$ as

$$
\eta(k) \triangleq y(k) \operatorname{Sin}(\hat{\Theta}(k))+z(k) \operatorname{Cos}(\hat{\Theta}(k))
$$

which may be rewritten as

$$
\begin{aligned}
\eta(k) & =A \operatorname{Cos}(\tilde{\Theta}(k))+n_{Q}(k) \\
n_{Q}(k) & =v_{i}(k) \operatorname{Sin} \hat{\Theta}(k)+v_{q}(k) \operatorname{Cos} \hat{\Theta}(k)
\end{aligned}
$$

From the observation that $\left\{v_{i}(k), v_{q}(k), \omega(k)\right\}$ forms an independent sequence and the fact that $\hat{\Theta}(k)$ is measurable with respect to the noise samples up to time $k-1$, it follows that

$$
E\left[n_{I}(k)\right]=0 \quad ; \quad E\left[n_{Q}(k)\right]=0 \quad, \quad E\left[n_{I}^{2}\right]=E\left[n_{Q}^{2}\right]=E\left[n_{Q}^{2}\right]=r \quad, \quad E\left[n_{I}(k) n_{Q}(k)\right]=0
$$

Thus $n_{I}(k)$ and $n_{Q}(k)$ are independent. Strictly speaking $\left\{n_{I}(k)\right\}$ or $\left\{n_{Q}(k)\right\}$ is not an independent sequence as $n_{I}(k)$ is correlated to $n_{I}(k-1)$ via $\hat{\Theta}(k)$. However, such a correlation is negligible* and is generally ignored in Phase Locked Loop Theory [16].

Thus we observe that an independent observation is available and exploit it in the following manner to improve the estimate of $x(k)$.

Among several possible methods, we select an iterative method wherein the measurements $\{y(k), z(k)\}$ are divided into groups of $M$ measurements with $M$ equal to $N$ or an integer submultiple of $N$. Each of these groups is then processed by the estimation algorithm 2 or more times. Considering the first such group with $\mathrm{k}$ between 1 and $\mathrm{M}$, then under low signal-to-noise ratio (SNR) condition, the estimate of total

* Essentially $\tilde{\Theta}(k)$ is negligible compared to $\Theta(k)$ and thus $\hat{\Theta}(k)$ in the expressions for $n_{I}(k), n_{Q}(k)$ may be replaced by $\Theta(k)$ which is a process independent of the additive noise process $\left\{v_{i}(k), v_{q}(k)\right\}$ 
phase $\Theta(k)$ in the first iteration denoted by $\hat{\Theta}^{1}(k)$ would be considerably different from its estimate $\hat{\Theta}^{2}(k)$ in the second iteration, i.e. $\left|\hat{\Theta}^{2}(k)-\Theta(k)\right|<<\left|\hat{\Theta}^{1}(k)-\Theta(k)\right|$. It is easily seen that the corresponding noise samples appearing in the prediction error term in (18) and denoted by $n_{I}^{1}(k), n_{I}^{2}(k)$ respectively have a correlation coefficient equal to $\operatorname{Cos}\left(\hat{\Theta}^{2}(k)-\hat{\Theta}^{1}(k)\right)$. Thus if the difference $\hat{\Theta}^{2}(k)-\hat{\Theta}^{1}(k)$ is approximately equal to $\pi / 2$, the corresponding noise samples are nearly independent and a signal processing gain of about $3 \mathrm{~dB}$ is realized. This effectively corresponds to using a prediction error $\eta(k)$ given by (19), in the second recursion. For a given signal-to-noise ratio, the value of $\mathbf{M}$ can be optimized to achieve maximum possible improvement. Note however, that no such gain can be realized under high SNR case as under such conditions $\hat{\Theta}^{1}(k) \cong \Theta(k) \cong \hat{\Theta}^{2}(k)$. These observations have been verified by computer simulations. It may be stated that improvement here is not due to iterative linearization of the nonlinearity as is usually the case but rather due to very specific properties of the noise $n_{I}(k)$.

Remarks: 1 . As is usually the case, $K(k+1)$ in (15) being independent of measurements may be precomputed and can possibly be replaced by its steady-state value.

2. Here we have worked with a complex baseband signal. However, similar ideas can be applied to scalar sampling case $[3,11]$ where a Phase-Locked Loop structure results.

\section{Modified Phase Locked Loop with up to $3 \mathrm{~dB}$ Improvement}

In this section we also provide an explicit implementation in terms of a phase-locked loop (PLL), see [3, 11] for details. Such an implementation is shown in Figure 3, where to keep consistency with the notation of previous sections, we represent both the received bandpass signal $s(k+1)$ and the NCO (numerically controlled oscillator) signal in terms of the same frequency $\omega_{c}$. Any difference can of course be absorbed in $\omega_{0}$, a part of the state vector. All other symbols in the figure are consistent with the rest of the publication except that $x(k)$ and $\Phi$ would correspond to their use in [11]; i.e. $x(k)$ here consists of instantaneous phase, frequency, etc. rather than the values of these at some fixed time instance. Note also that the bandpass noise $\bar{v}(k+1)$ of Figure 3 has the following quadrature representation in terms of $v_{i}(k)$ and $v_{q}(k)$ of $(3)$.

$$
\bar{v}(k)=v_{i}(k) \operatorname{Sin}\left(\omega_{c} t_{k}\right)+v_{q}(k) \operatorname{Cos}\left(\omega_{c} t_{k}\right)
$$

The loop filter may be designed either on the basis of steady-state solution of the Riccati equation (Kalman 
Gain) or via any other design procedure applicable to PLL filter design.

To see the possible $3 \mathrm{~dB}$ improvement due to Hilbert transform, simply note that if $\tilde{\Theta}((k)$ is equal to $k T_{s} \tilde{\omega}$ for some frequency error $\tilde{\omega}$ with $\eta(k)$ given by

$$
\eta(k)=A \operatorname{Cos}\left(\tilde{\omega} k T_{s}\right)+n_{Q}(k)
$$

then its Hilbert transform is simply

$$
\begin{aligned}
\hat{\eta}(k) & =A \operatorname{Sin}\left(\tilde{\omega} k T_{s}\right)+\hat{n}_{Q}(k) \\
& =A \operatorname{Sin}(\tilde{\Theta}(k))+\hat{n}_{Q}(k)
\end{aligned}
$$

Moreover, the independence of $n_{I}(k)$ and $n_{Q}(k)$ also implies the independence of $n_{I}(k)$ and $\hat{n}_{Q}(k)$ due to Gaussian assumption on noise and the fact that the Hilbert transform is a deterministic linear transform. The fact that $\tilde{\omega}$ is actually time-varying is taken into account by making the Hilbert transform adaptive, i.e. in time-domain we replace the Hilbert transform by its following adaptive discrete time version;

$$
\hat{\eta}(k)=\frac{T_{s}}{\pi} \sum_{j} \frac{\lambda^{k-j}}{(k-j)} \eta(j)
$$

where $\lambda$ is some adaptation constant with $0<\lambda<1$. For $\lambda=1$, (23) corresponds to the discrete time version of the standard continuous-time convolution definition (24).

$$
\hat{\eta}(t)=\frac{1}{\pi} \int_{\tau} \frac{1}{(t-\tau)} \eta(\tau) d \tau
$$

The parameter $\lambda$ is selected so that over an interval $T_{s} /(1-\lambda)$, the frequency $\tilde{\omega}$ remains nearly constant. Thus the selection of $\lambda$ depends upon the input signal dynamics, loop bandwidth, etc. It may be noted that the implementation of Hilbert transform by necessity requires a delay in the loop and thus the actual realized gain may be less than $3 \mathrm{~dB}$ depending upon the amount of such a delay. 


\section{SIMULATIONS}

In the following we present simulation results obtained when the algorithm is applied to the signal parameters acquisition and tracking for a high dynamic GPS receiver [7, 12]. For the purposes of this simulation we assume that the pseudo random code has been removed from the received signal and symbol timing has been acquired. Such a signal is then quadrature sampled at a rate that is an integer multiple $\mathrm{N}$ of the bit rate $1 / T_{b}$, the latter assumed to be $50 \mathrm{bps}$. For the purposes of simulations, we consider two different sampling rates of 750 and 1000 samples/sec corresponding to the value of $\mathrm{N}$ equal to 15 and 20 samples/bit respectively. The simulation results are presented for different values of $P / N_{0}$, where $\mathrm{P}$ denotes the received carrier power and $N_{0}$ is the one-sided power spectral density of the receiver bandpass noise. The parameters of equivalent baseband model (4) are then related as

$$
\frac{A^{2}}{2 \sigma^{2}}=\frac{1}{N}\left(\frac{P T_{b}}{N_{0}}\right) \quad ; \quad \sigma^{2}=E\left[v_{i}^{2}\right]
$$

with $\frac{P}{N_{0}}(\mathrm{~dB})=\frac{P T_{\mathrm{b}}}{N_{0}}(\mathrm{~dB})+17$. For the purposes of simulations we use normalized parameters as discussed in $[8,9]$. The performance of the algorithm is completely invariant to such a simple transformation, but in implementation such a normalization can be very convenient as it keeps the condition number of the error covariance matrix small, and thus the algorithm would be more robust under finite precision arithmetic.

The normalized sampling period $T_{s N}$ is selected to be $.02 \mathrm{~s}$ with corresponding normalized parameters $\omega_{0 N}, \gamma_{0 N}$ and $\delta_{0 N}$ expressed in units of rad/sec. The parameters of interest i.e., the receiver velocity $v_{d}$ in $\mathrm{m} / \mathrm{s}$ and its derivatives in appropriate units can be expressed in terms of the above normalized parameters via

$$
\begin{aligned}
v_{d} & =\frac{N \omega_{0 N}}{2 \pi} \frac{c}{f_{c}} \\
a & =\dot{v}_{d}=2 \frac{N^{2} \gamma_{0 N}}{2 \pi} \frac{c}{f_{c}} \\
J & =\ddot{v}_{d}=6 \frac{N^{3} \delta_{0 N}}{2 \pi} \frac{c}{f_{c}}
\end{aligned}
$$

where $c$ is the velocity of light and $f_{c}$ is the received carrier nominal frequency equal to $1.575 \mathrm{GHz}$. Figures 4-7 plot both the estimates of the normalized parameters as well as those of the receiver physical parameters $v_{d}, a$ and $J$ defined in (26). We consider first the acquisition problem where in the initial parameter vector 
$\psi_{0}$ is some unknown constant. The modification to include the time-varying case is subsequently considered.

For the simulation results presented below, a time-varying exponential data weighting factor given by (A7) is used with different values for the parameters $\lambda_{0}, \lambda(0)$ and $\lambda_{\max }$ for the "global" parameter and "local" parameter estimation purposes. The values of these parameters used in simulations are specified below.

$\begin{array}{lccc} & \lambda_{0} & \lambda(0) & \lambda_{\max } \\ \text { "Local" Estimator } & 1.0 & .99 & .99 \\ \text { "Global" Estimator } & .995 & .99 & .998\end{array}$

Note that for $\lambda_{0}<1$, the value of $\lambda(k)$ when computed according to (A7), it monotonically increases to 1 as $k \rightarrow \infty$. In the simulations if $\lambda\left(k_{0}\right) \geq \lambda_{\max }$ for some $k_{0}$, we simply set $\lambda(k)=\lambda_{\max }$ for $k \geq k_{0}$.

Considering the estimation of "local" parameters, it is apparent that there is much more uncertainty in the phase parameter $\theta\left(T_{b}+\right)$ compared to other parameters (a good prediction of the other parameters is available from "global" parameter estimates). This fact was taken into account to marginally improve the performance by multiplying the $(1,1)$ element of the matrix $\lambda^{-1}(k) \Phi P(k / k) \Phi^{\prime}$ in $(\mathrm{A} 5)$ by $\lambda(k) / \bar{\lambda}_{1}$ with $\bar{\lambda}_{1}$ $=\min \left(\lambda_{1}, \lambda(k)\right)$. In the simulations $\lambda_{1}$ is equal to 0.9 .

A diagonal initial covariance matrix $P_{G}(0 /-1)$ (G denotes "global") for the "global" estimation algorithm is selected with its diagonal elements equal to $10^{6}, 10^{6}, 10^{3}$, reflecting the initial uncertainty about the parameters. The corresponding initial covariances for the local estimations are simply given by $S\left(m T_{b}\right) P_{G}(m N / m N) S\left(m T_{b}\right)$ where $S\left(m T_{b}\right)$ is a transformation similar to $S\left(T_{b}\right)$ of $(6)$, and $P_{G}(m N / m N)$ denotes the filter error covariance matrix for the "global" parameter estimator after $m N$ measurements. A diagonal $Q(k)$ matrix in (A5) is used with all its elements equal to zero except $(1,1)$ element which is equal to 0.5 if $k$ is an integer multiple of $N$ in the global algorithm, or for $k=1$ in the "local" algorithm to take into account phase noise due to data detection errors. Note that the extent of the "local" algorithms is confined to 1 bit of duration and thus the value of $\lambda(k)$ is reset to $\lambda(0)$ at the start of each new bit for fast convergence.

Figures 4-7 present the results of typical simulation runs for the case when an unknown constant acceleration and unknown phase and frequency are introduced and a third order model for the state vector is 
used. In the following we present results for $\hat{\omega}_{F}\left(k T_{b}-\right)$ and $\hat{\gamma}_{F}\left(k T_{b}+\right)$ obtained in step 2 of the algorithm along with $\hat{\bar{\theta}}\left(k T_{b}+\right)$ of step 3 . Figures 4 and 5 present the simulation results for the case of $22.8 \mathrm{~dB}-\mathrm{Hz}$ with the unknown acceleration of $50 \mathrm{~g}$. Also, a randomly selected unknown phase of -2.3 rad. along with an unknown velocity error of about $25 \mathrm{~m} / \mathrm{s}$ was included in the simulations. Such an error is expected when the algorithm is extended for tracking. In figure 4 we consider the case of no data detection (data bits assumed known). As is apparent from figures $4 b$ and c, good estimates of both the acceleration and velocity are obtained within a period of about 10 data bits $(200 \mathrm{~ms})$. In this period the velocity error is reduced to about $0.2 \mathrm{~m} / \mathrm{s}$ with acceleration error of about $2 \mathrm{~m} / \mathrm{s}^{2}$ in various simulation runs.

In figure 4c, the phase estimation error at the start of bit periods is plotted. Similar results are obtained for the phase estimates just before the data transitions at time $k T_{b}-$. From the drift in phase estimates as seen in the figure, it is clear that at the low CNR ratio considered here, phase coherence is maintained over short periods. However, such coherence is adequate for the differential detection discussed in the publication. In the simulation reported, six errors occurred over a period of about 40 bits. This may be compared with a DPSK detection (constant frequency) which results in a probability of error $P_{e}$ of about .06 at $\left(P T_{b} / N_{0}\right)$ of $6 \mathrm{~dB}$, and with coherent PSK detection (coherent phase) which results in $P_{e}$ of .01 [14]. Here, as the received signal frequency is time varying, the probability of error is even higher as may be expected. However, even under such a poor detection probability, good estimates of frequency and frequency rate can be achieved as shown in figure 5 .

In figure $5 b, c$, the estimates of velocity and acceleration are plotted for the case when data is detected simultaneously with parameter estimation. Compared to figure $4 b, c$ the estimation errors are considerably higher. However, in a period of about $200 \mathrm{~ms}$, velocity and acceleration estimation errors reduce to only about $1 \mathrm{~m} / \mathrm{s}$ and $15 \mathrm{~m} / \mathrm{s}^{2}$ respectively, which are quite small.

In figures 6 and 7 we present the estimation errors for the case when the sampling rate is equal to 20 samples/bit. Keeping the sample SNR $\left(A^{2} / 2 \sigma^{2}\right)$ the same as for figure 1 , this corresponds to a $\left(P / N_{0}\right)$ to $24.1 \mathrm{~dB}-\mathrm{Hz}$. Comparison of figures 4 and 7 shows that the sampling rate does not significantly affect the estimation errors. Figure 7 presents the results for the case of $100 \mathrm{~g}$ step acceleration. 


\section{CONCLUSIONS}

We have presented a novel simultaneous estimation-detection scheme for the efficient estimation of phase, frequency and frequency derivative of a sinusoidal signal which may possibly be phase modulated by binary data and is received under noise. The proposed scheme is of a very general nature and can easily be adapted to situations other than those considered in the publication. For example, the scheme is equally applicable to situations where the data is QPSK on FSK (Frequency Shift Keying) modulated. The proposed algorithm is recursive in the number of data bits and within a bit period it is recursive in the number of signal samples. In terms of its application to GPS receivers, we observe from simulations, some of which have been reported here, that for a $P / N_{0}$ of $23-24 \mathrm{~dB}-\mathrm{Hz}$, and with a $50-100 \mathrm{~g}$ acceleration, the scheme is capable of acquiring the velocity with an error of about $1 \mathrm{~m} / \mathrm{s}$ in a $200 \mathrm{~ms}$ period. Thus, the algorithm can easily be adapted to high dynamic tracking situations as well. The most straightforward adaptation is simply to restart the algorithm every $200 \mathrm{~ms}$. In fact, since the acceleration does not change by more than $20 \mathrm{~g}$ (even for a $100 \mathrm{~g} / \mathrm{s}$ jerk) over a $200 \mathrm{~ms}$ period, the actual performance could be expected to be better for tracking of $100 \mathrm{~g} / \mathrm{s}$ jerk than reported here. Results of [7], which also consider data modulated carrier, show that an error of the order of $1 \mathrm{~m} / \mathrm{s}$ is obtained for a $P / N_{0}$ of about $30 \mathrm{~dB}-\mathrm{Hz}$ under similar dynamics. From this comparison it is apparent that the algorithm can achieve a very significant improvement of $6 \mathrm{~dB}$ over a previously proposed scheme based on Fast Fourier Transform (FFT) techniques. More extensive simulations of the proposed algorithm would provide a more precise measure of such an improvement. 


\section{REFERENCES}

[1] Rife, D.C., "Single Tone Parameter Estimation from the Discrete-Time Observations," IEEE Transactions on Information Theory, Vol. IT-20, No. 5, pp. 591-598, September 1974.

[2] Friedland, B., "Optimum Steady-State Positions and Velocity Estimation Using Sampled Position Data," IEEE Transactions on Aerospace and Electronic Systems, Vol. 9, pp. 906-911, November 1973.

[3] Polak, D.R., Gupta, S.C., "Quasi Optimum Digital Phase-Locked Loops," IEEE Transactions on Communications, Vol. 21, pp. 75-82, January 1973.

[4] Tufts, D.W., Kumaresan, R., "Estimation of Frequencies of Multiple Sinusoids: Making Linear Prediction Perform Like Maximum Likelihood," Proceedings of the IEEE, Vol. 70, No. 9, pp. 975-987, September 1982.

[5] Friedlander, B., "On the Cramer-Rao Bound for Time Delay and Doppler Estimation," IEEE Transactions on Information Theory, Vol. IT-30, No. 3, pp. 575-580, May 1984.

[6] Abatzoglou, T.J., "A Fast Maximum Likelihood Algorithm for Frequency Estimation of a Sinusoid Based on Newton's Method," IEEE Transactions on Acoustics, Speech and Signal Processing, Vol. ASSP-33, No. 1, pp. 77-89, February 1985.

[7] Hurd, W.J., Statman, J.I. and Vilnrotter, V.A., "High Dynamic GPS Receiver Using Maximum Likelihood Estimation and Frequency Tracking," IEEE Trans. AES. Vol. 23, No. 5, pp. 425-437, September 1987.

[8] Kumar, R., "Fast Frequency Acquisition via Adaptive Least Squares Algorithm," Proceedings of the International Telemetering Conference, Las Vegas, pp. 91-101, October 1986.

[9] Kumar, R., "Differential Sampling for Fast Frequency Acquisition via Adaptive Extended Least Squares Algorithm," Proceedings of the International Telemetering conference, San Diego, pp. 191-201, October 1987.

[10] Kumar, R. and Hurd, W.J., "A Class of Optimum Digital Phase-Locked Loops," Proceedings of the 25th IEEE Conference on Decision and Control, December 1986, Athens, Greece.

[11] Kumar, R. and Hurd, W.J., "Fixed Lag Smoothers for Carrier Phase and Frequency Tracking," Proceedings of the IASTED International Symposium on Applied Control and Identification, pp. 160-166, 
December 1986, also submitted to IASTED Journal. See also, "Optimum Filters and Smoothers Design for Carrier Phase and Frequency Tracking," JPL Publication 87-10, National Aeronautics and Space Administration, Jet Propulsion Laboratory, California Institute of Technology, Pasadena, California.

[12] Vilnrotter, V.A., Hinedi, S. and Kumar, R., “A Comparison of Frequency Estimation Techniques for High Dynamic Trajectories," JPL Publication 88-21, Jet Propulsion Laboratory, California Institute of Technology, Pasadena, California.

[13] Hoefener, C.E. and Wells, L., "Utilizing GPS for Ultra-High Dynamic Vehicle Tracking in Space," Proceedings of the International Telemetering Conference, Las Vegas, pp. 771-773, October 1986.

[14] Couch, L.W., Digital and Analog Communication Systems, Macmillan, New York, 1986.

[15] Lindsey, W.C. and Simon, M.K., Telecommunications Systems Engineering, Prentice-Hall, 1973.

[16] Anderson, B.D.O. and Moore, J.B., Optimal Filtering, Prentice-Hall, 1979. 


\section{A PPENDIX}

Denoting by $\hat{x}(k / j)$ the estimate of $x(k)$ on the basis of observations up to time $j$, one obtains $[11,16]$ Filter State Equations:

$$
\begin{gathered}
\hat{x}(k+1 / k+1)=\Phi \hat{x}(k / k)+M(k+1) \nu(k+1) \\
\nu(k+1)=z(k+1)-h(\hat{x}(k+1 / k), k+1)
\end{gathered}
$$

Filter Gain:

$$
M(k+1)=P(k+1 / k) h_{x}^{\prime}(\hat{x}(k+1 / k), k+1) \sigma^{-1}(k+1)
$$

Error Covariance Update:

In (A3) $P(k / j)$ denotes the error covariance matrix $E\left[\{x(k)-\hat{x}(k / j)\}\{x(k)-\hat{x}(k / j)\}^{\prime}\right]$, with a recursive update given by

$$
\begin{gathered}
P(k+1 / k+1)=P(k+1 / k)-P(k+1 / k) h_{x}^{\prime} \sigma^{-1}(k+1)\left[P(k+1 / k) h_{x}^{\prime}\right]^{\prime} \\
P(k+1 / k)=\lambda^{-1}(k) \Phi P(k / k) \Phi^{\prime}+Q(k) \\
\sigma(k+1)=h_{x} P(k+1 / k) h_{x}^{\prime}+R \\
\lambda(k+1)=\lambda_{0} \lambda(k)+\left(1-\lambda_{0}\right) \quad ; \quad \lambda(k) \leq \lambda_{\max }
\end{gathered}
$$

where $\lambda(k)$ is the exponential data weighting coefficient, $\lambda_{0}$ is some non-zero positive number less than 1 , and $h_{x}$ denotes the derivative of the function $h$.

To simplify the algorithm above for the given signal model, one obtains from (15), 


$$
h_{x}(\hat{x}(k / k-1), k)=\left[\begin{array}{c}
A \operatorname{Cos}(\hat{\Theta}(k)) \\
-A \operatorname{Sin}(\hat{\Theta}(k))
\end{array}\right] J^{\prime}(k) \quad ; \quad \hat{\Theta}(k)=J^{\prime}(k) \hat{x}(k / k-1)
$$

Substitution of (A8) in (A6) results in

$$
\begin{gathered}
\sigma^{-1}(k)=\frac{1}{r^{2}+r a(k)}\left[\begin{array}{cc}
a(k) \operatorname{Sin}^{2}(\hat{\Theta}(k))+r & a(k) \operatorname{Cos}(\hat{\Theta}(k)) \operatorname{Sin}(\hat{\Theta}(k)) \\
a(k) \operatorname{Cos}(\hat{\Theta}(k)) \operatorname{Sin}(\hat{\Theta}(k)) & a(k) \operatorname{Cos}^{2}(\hat{\Theta}(k))+r
\end{array}\right] \\
a(k) \triangleq A^{2} J^{\prime}(k) P(k / k-1) J(k)
\end{gathered}
$$

Substitution of (A8) and (A9) in (A4) results in

$$
P(k+1 / k+1)=P(k+1 / k)-P(k+1 / k) J(k+1) A^{2}\left[J^{\prime}(k+1) P(k+1 / k) J(k+1)+r\right]^{-1} J^{\prime}(k+1) P(k+1 / k)
$$

The Kalman gain expression and the state update equations can similarly be simplified as

$$
\begin{gathered}
M(k+1)=A(a(k+1)+r)^{-1} J(k+1)[\operatorname{Cos}(\hat{\Theta}(k+1))-\operatorname{Sin}(\hat{\Theta}(k+1))] \\
\hat{x}(k+1 / k+1)=\Phi \hat{x}(k / k)+K(k+1) \in(k+1) \\
K(k+1)=\left[A^{2} J^{\prime}(k+1) P(k+1 / k) J(k+1)+r\right]^{-1} P(k+1 / k) A J(k+1) \\
\in(k+1)=y(k+1) \operatorname{Cos}(\hat{\Theta}(k+1))-z(k+1) \operatorname{Sin}(\hat{\Theta}(k+1))
\end{gathered}
$$

Equations (A12, (A5) and (A10) then constitute the extended Kalman filter equations for the signal model of interest. 

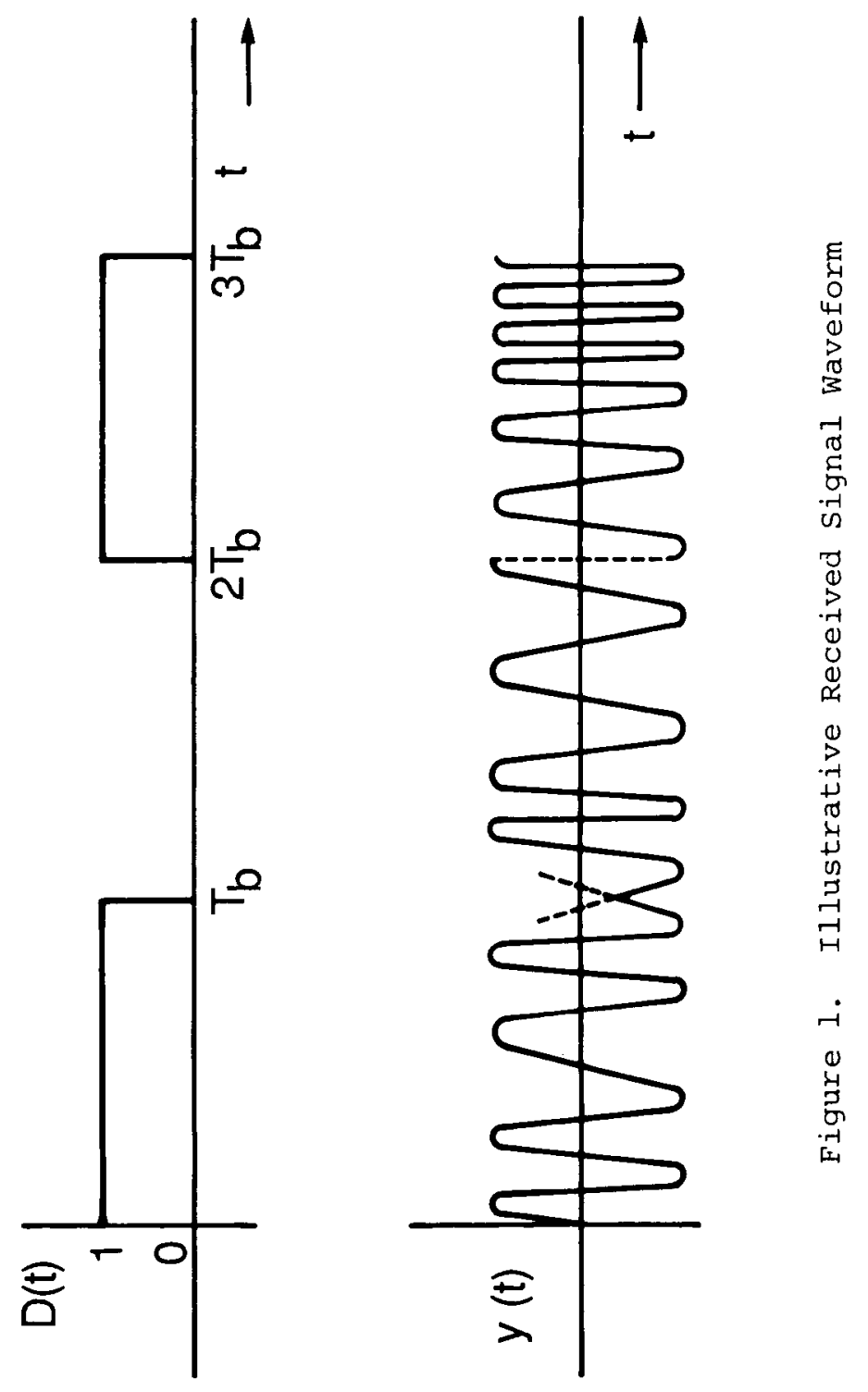


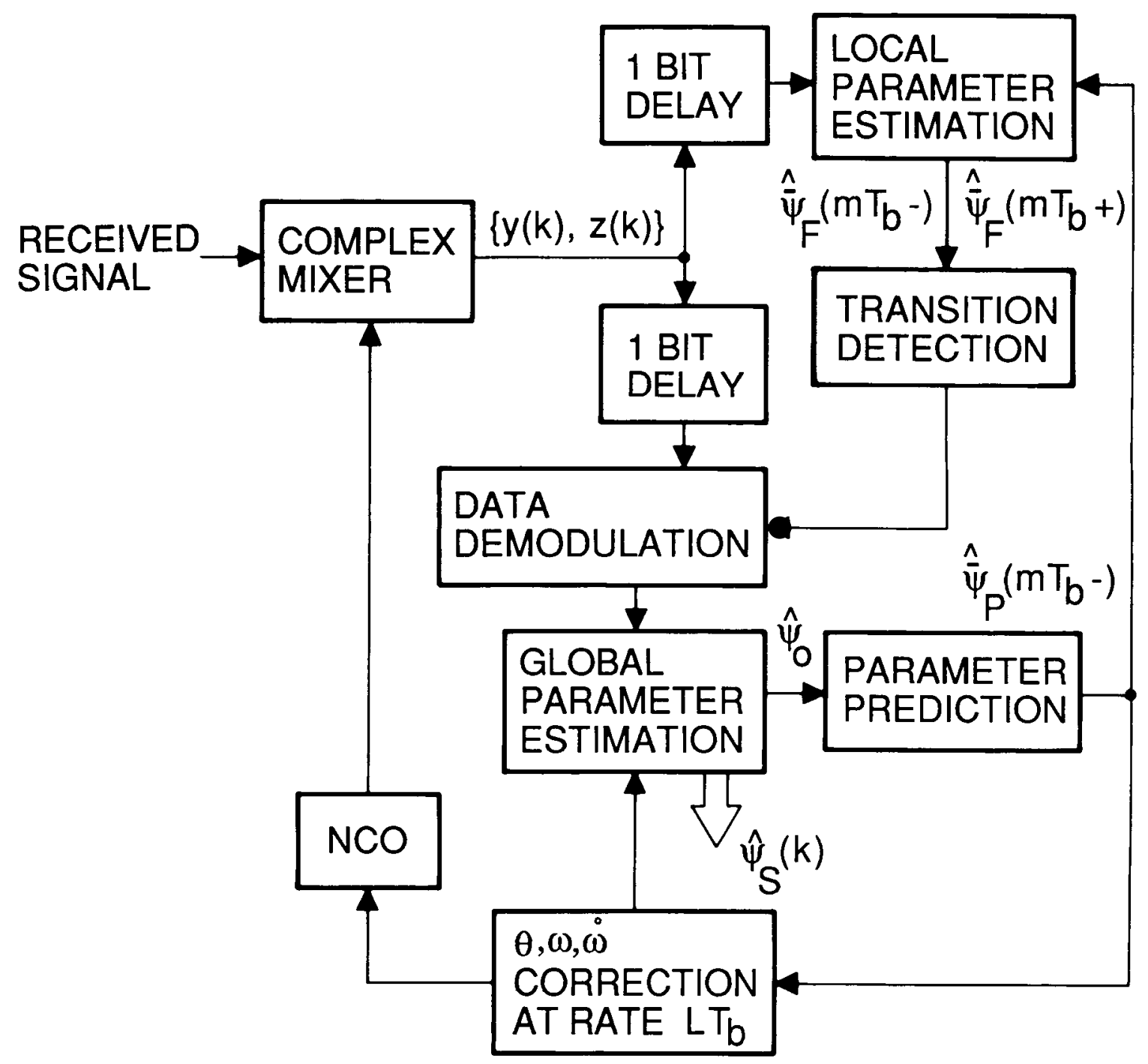

Figure 2. Schematic Diagram of the Proposed Estimation/ Detection Scheme 


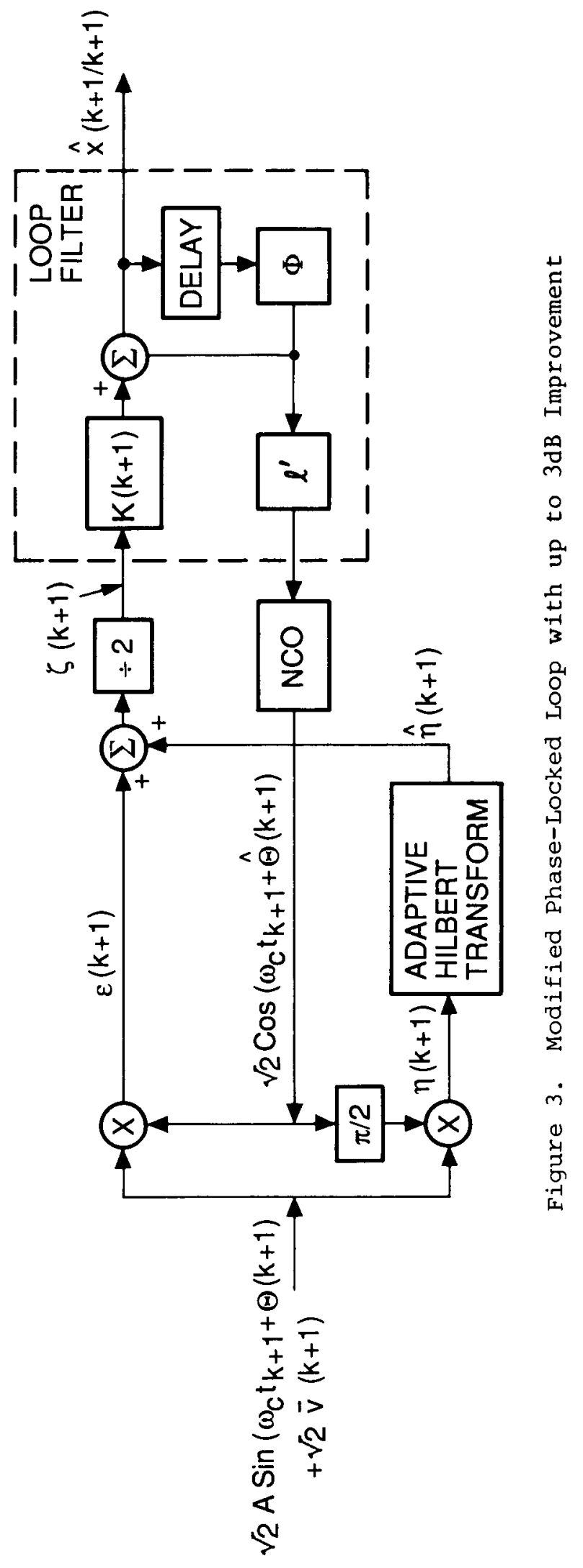




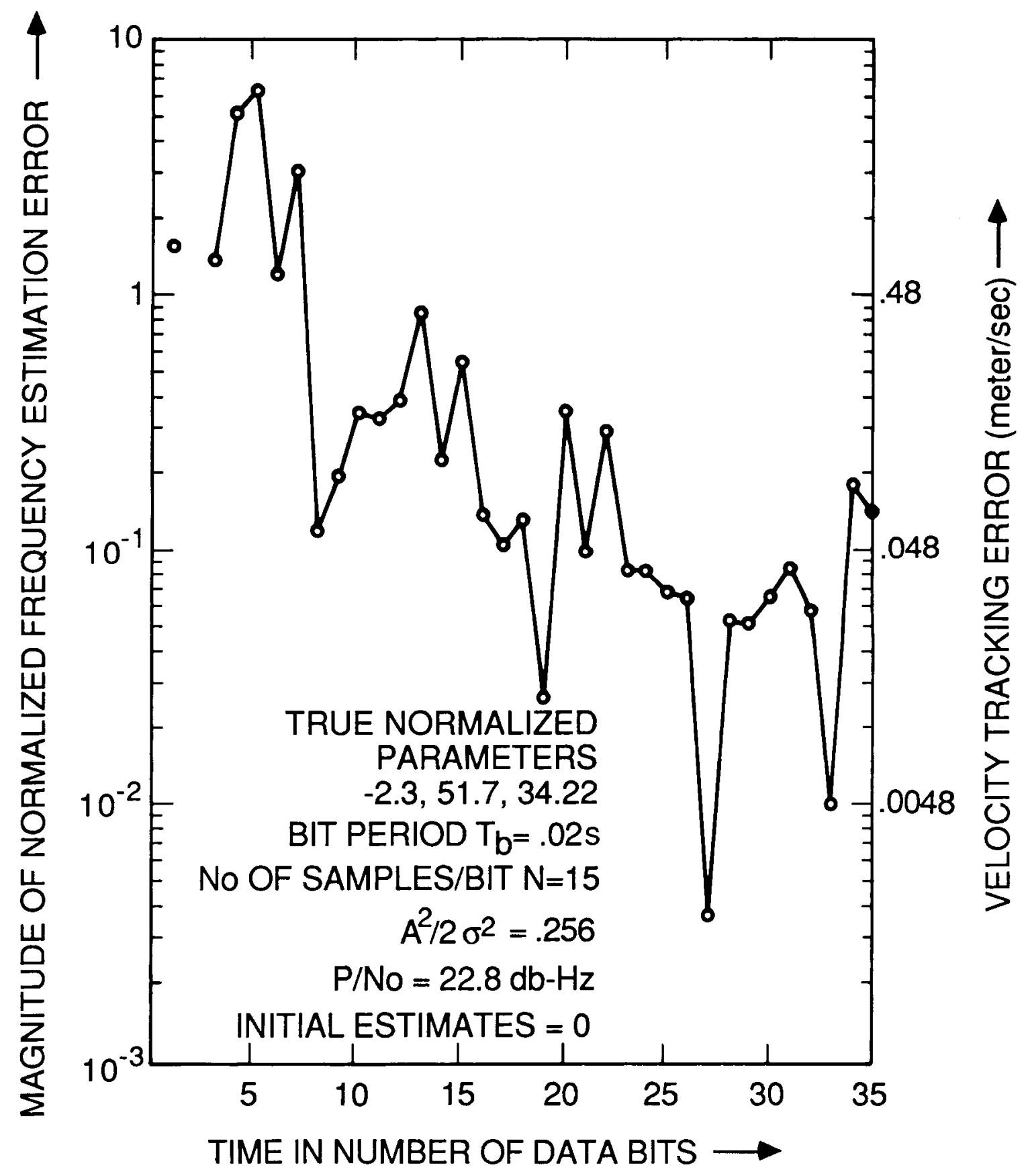

Figure 4a. Errors in Estimates of Frequency at Bit Intervals (Acceleration $50 \mathrm{~g}$ ) 


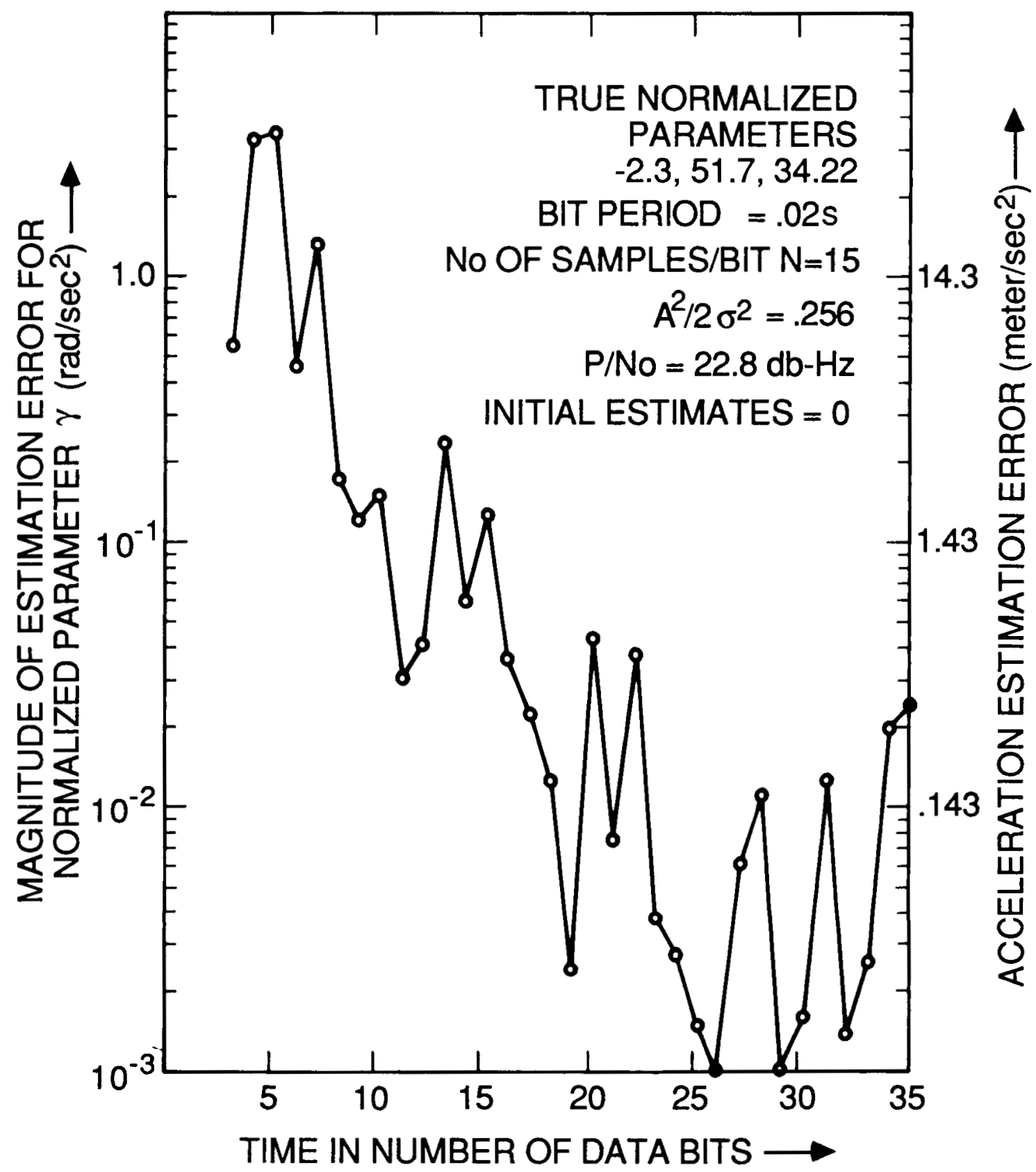

Figure 4b. Errors in Estimates of Frequency Derivative at Bit Intervals (Acceleration $50 \mathrm{~g}$ ) 


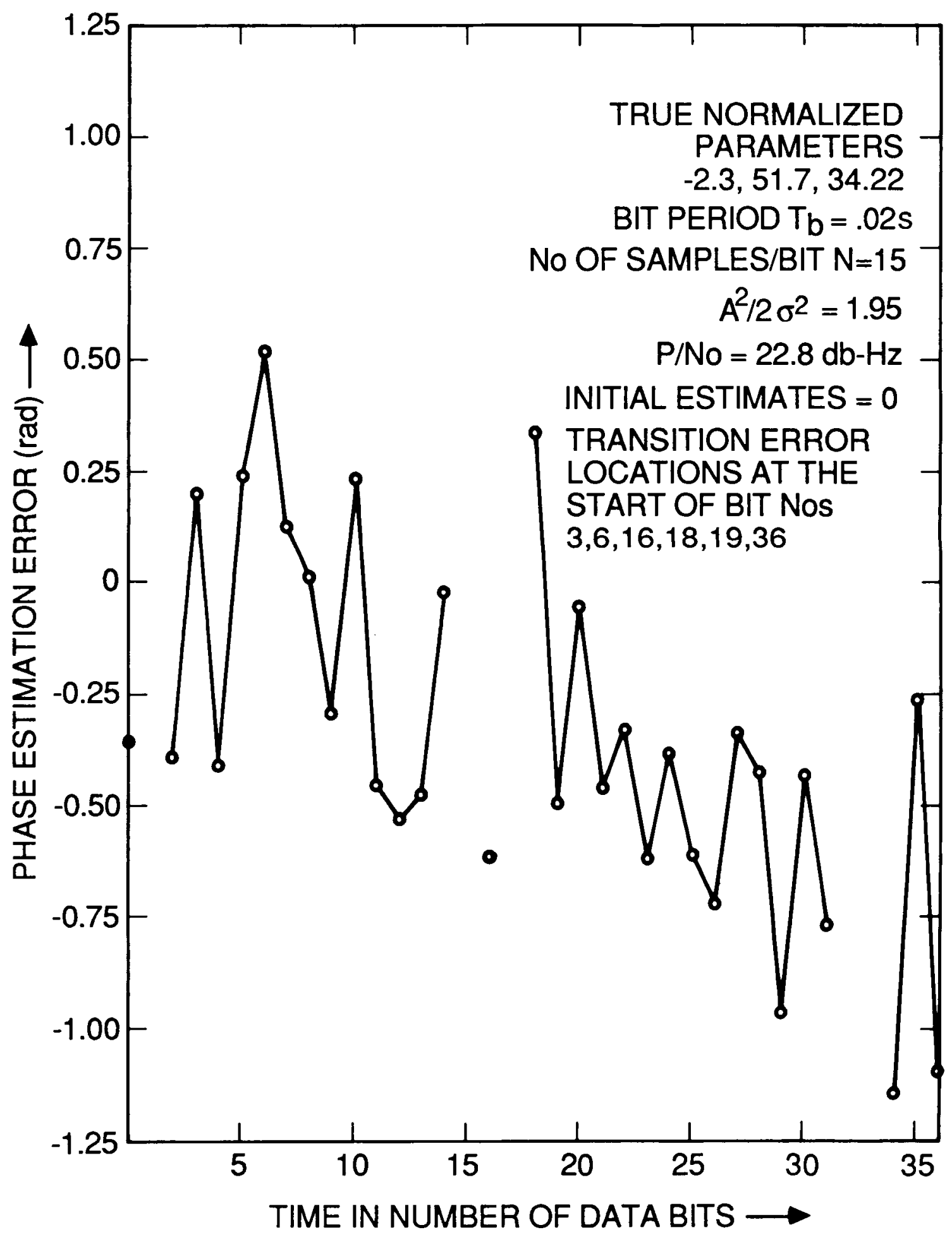

Figure 4c. Carrier Phase Estimation Error at the Start of Bit Periods (Acceleration 50g) 


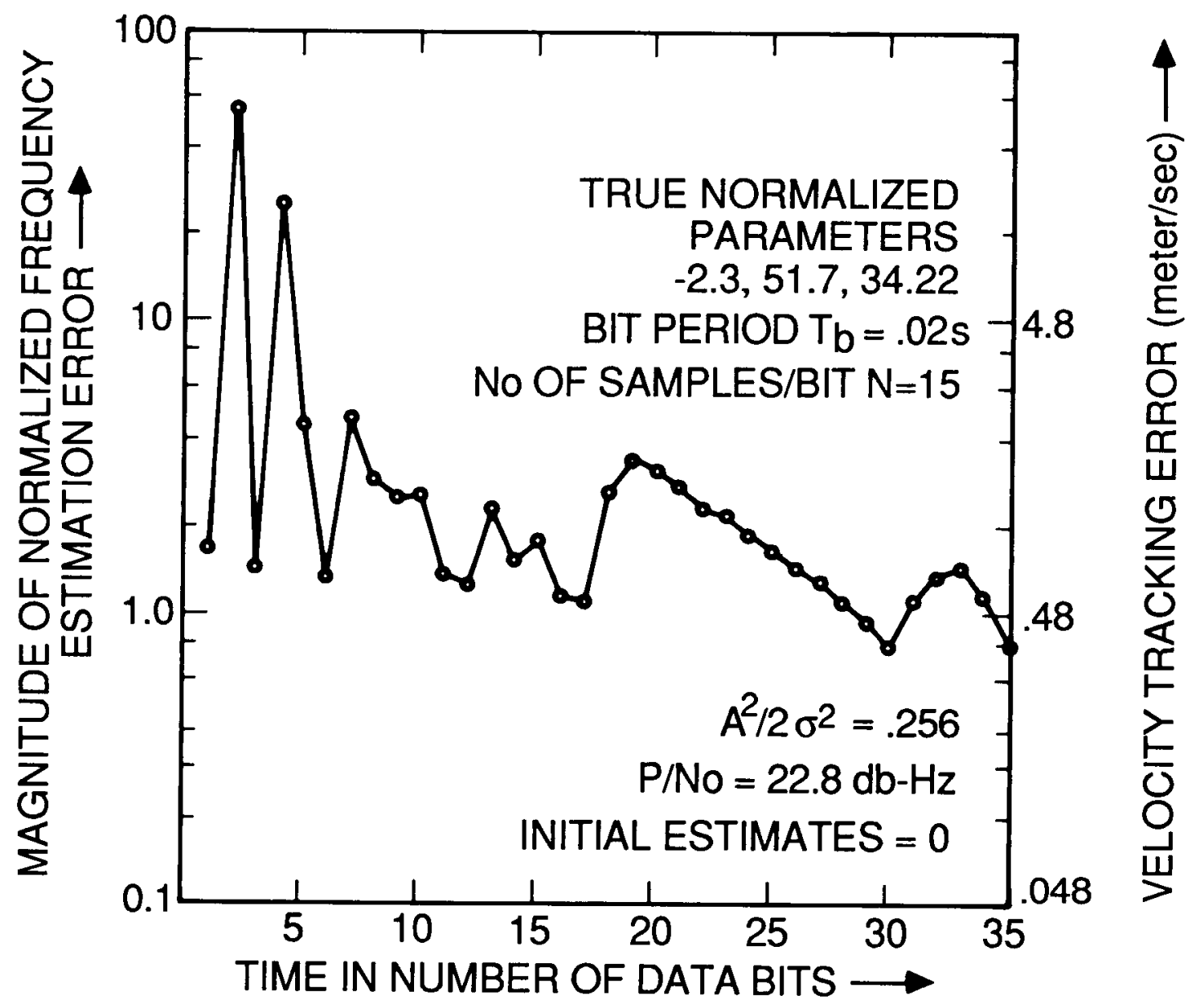

Figure 5a. Errors in Estimates of Frequency at Bit Intervals with Data Detection (Acceleration $50 \mathrm{~g}$ ) 


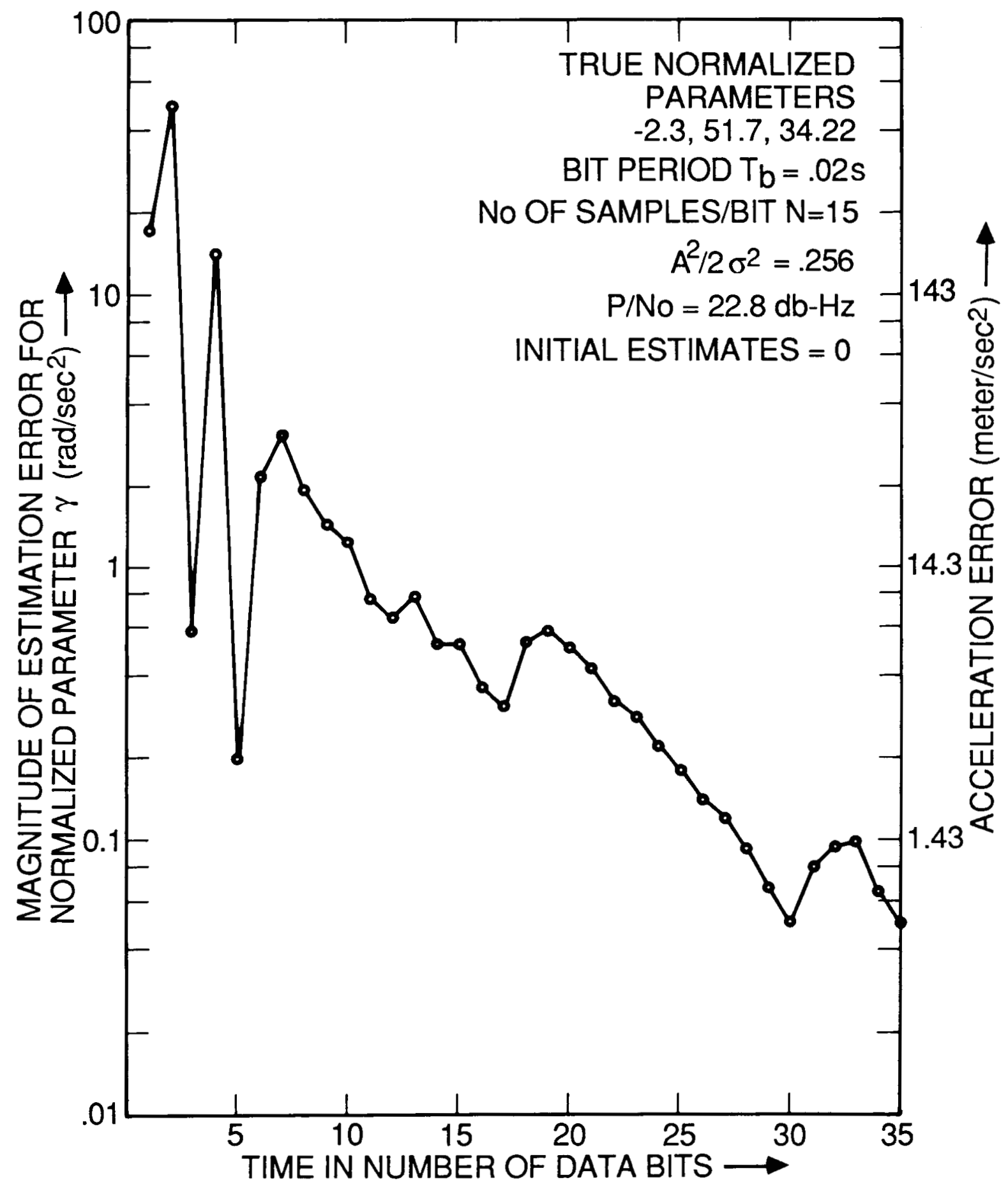

Figure 5b. Errors in Estimates of Frequency Derivative at Bit Intervals with Data Detection (Acceleration $50 \mathrm{~g}$ ) 


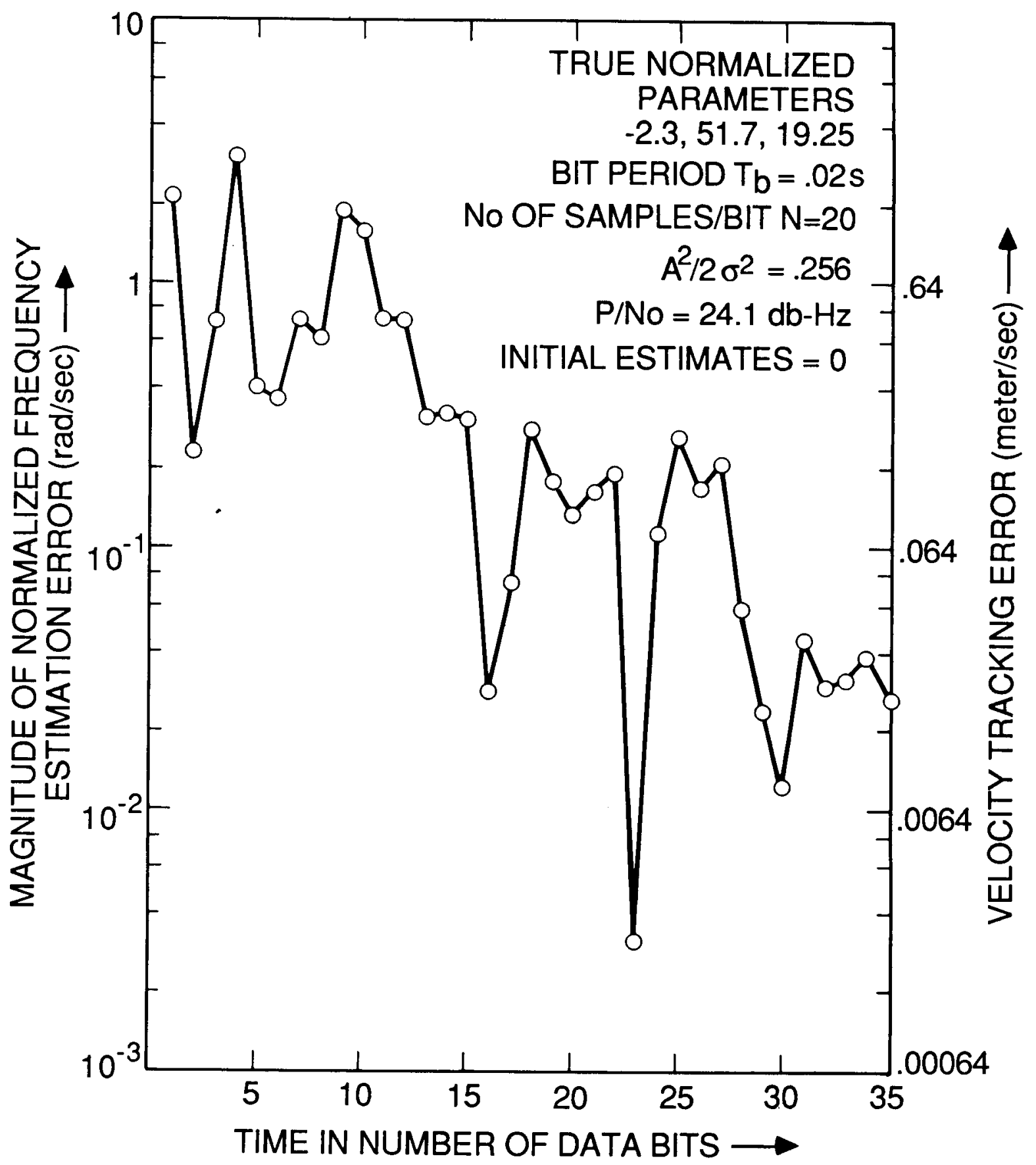

Figure 6a. Errors in Estimates of Frequency at Bit Intervals (Acceleration $50 \mathrm{~g}$ ) 


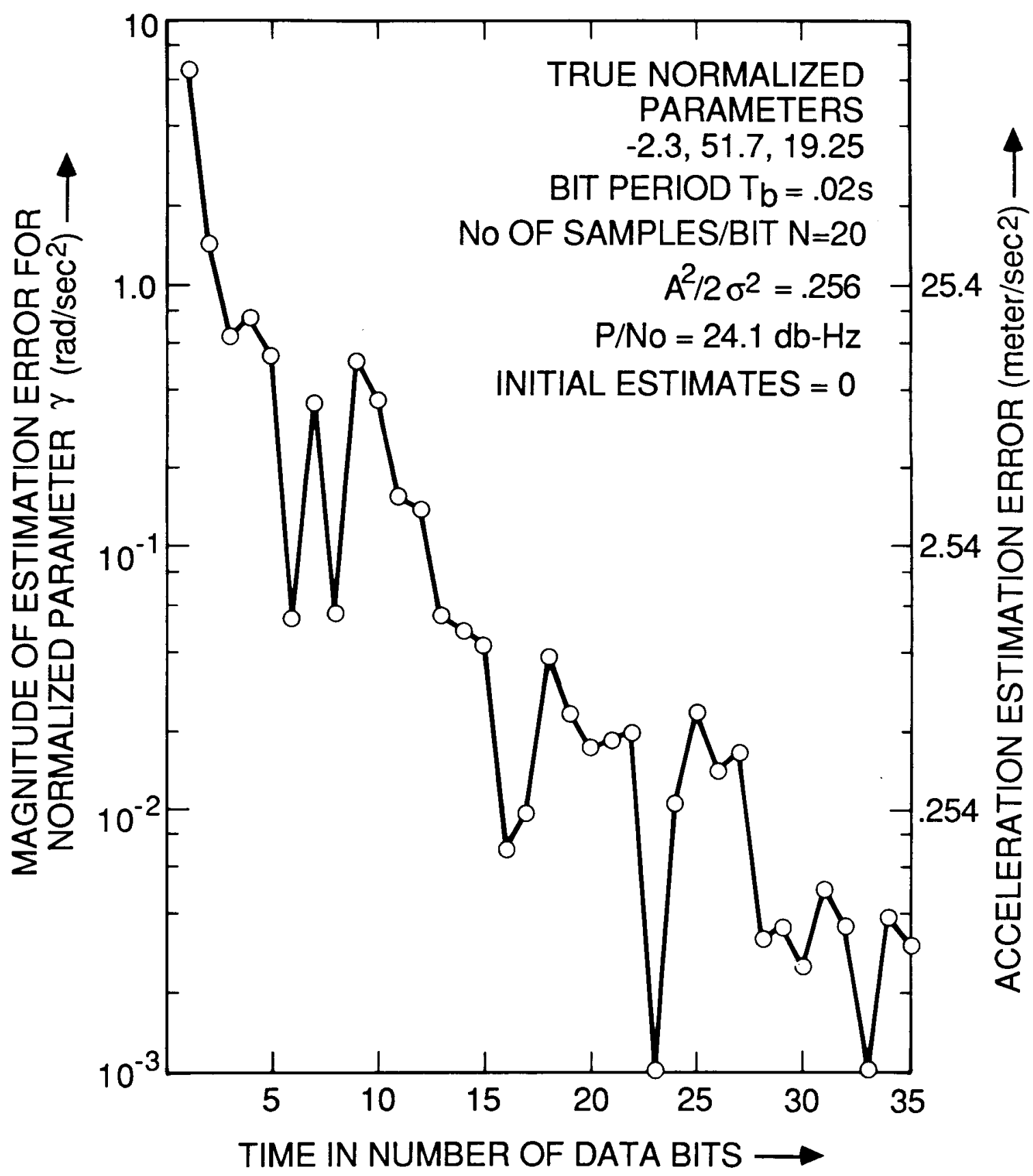

Figure 6b. Errors in Estimates of Frequency Derivative (Acceleration $50 \mathrm{~g}$ ) 


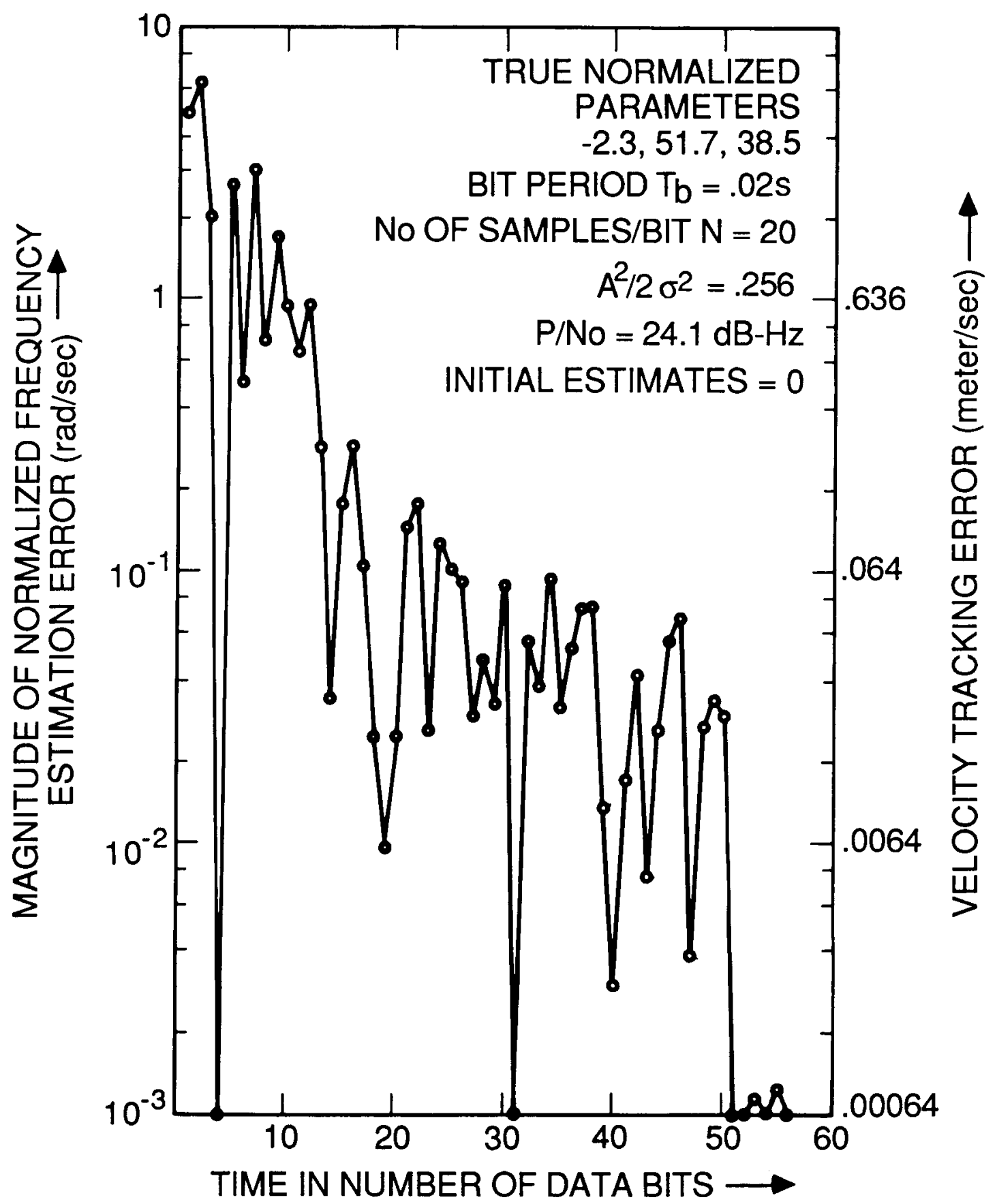

Figure 7a. Errors in Estimates of Frequency at Bit Intervals (Acceleration $100 \mathrm{~g}$ ) 


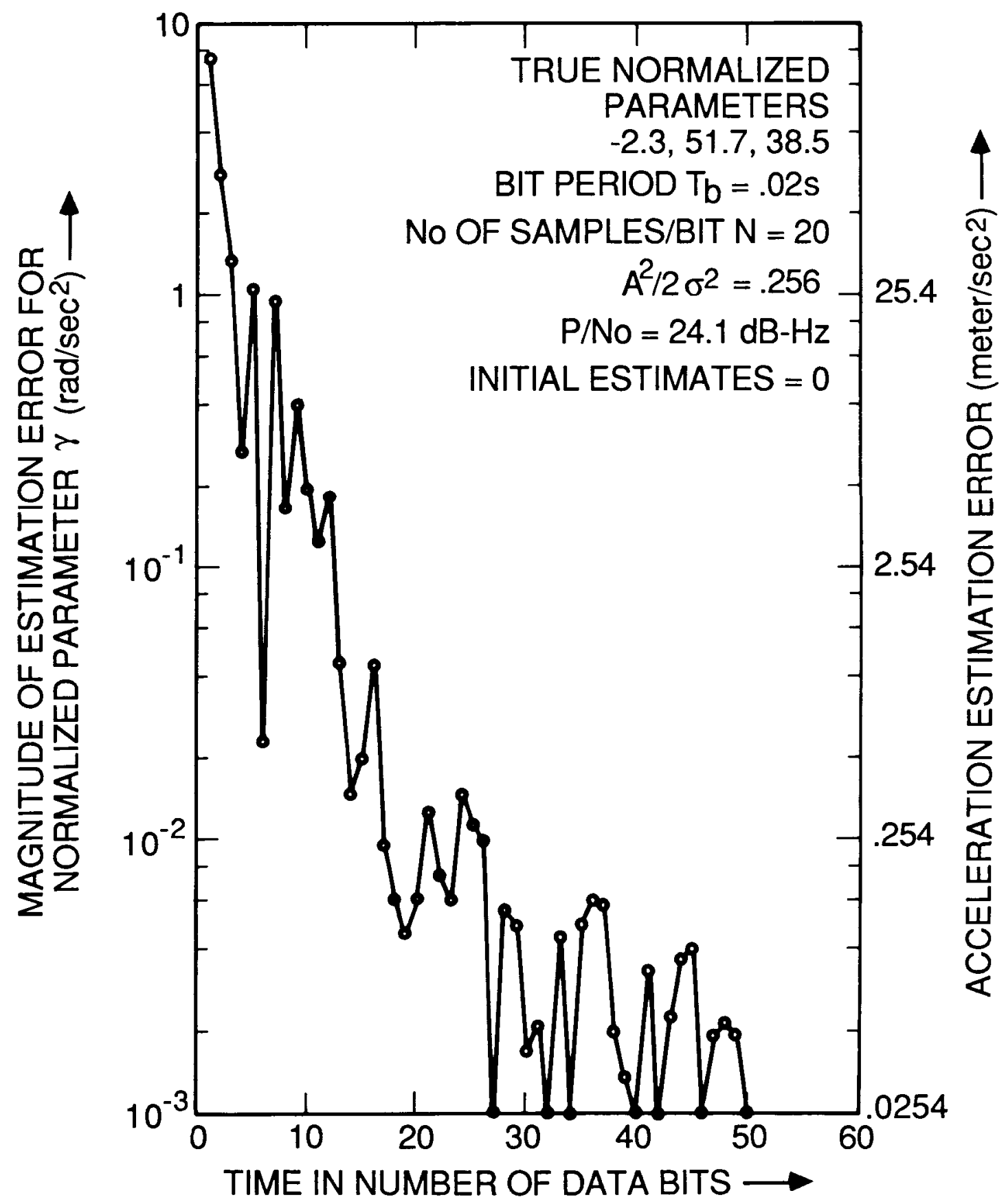

Figure 7b. Errors in Estimates of Frequency Derivative at Bit Intervals (Acceleration $100 \mathrm{~g}$ ) 
TECHNICAL REPORT STANDARD TITLE PAGE

\begin{tabular}{|c|c|}
\hline 2. Government Accossion No. & 3. Recipient's Catalog No. \\
\hline \multirow{2}{*}{$\begin{array}{l}\text { 4. Title and Subtitle } \\
\text { Efficient Detection and Signal Parameter Estimation } \\
\text { With Applications to High Dynamic GPS Receivers }\end{array}$} & $\begin{array}{l}\text { 5. Report Date } \\
\text { December 15, } 1988\end{array}$ \\
\hline & 6. Performing Organization Code \\
\hline $\begin{array}{l}\text { 7. Author(s) } \\
\text { R. Kumar }\end{array}$ & 8. Performing Organization Report No. \\
\hline \multirow{3}{*}{$\begin{array}{l}\text { 9. Performing Organization Name and Address } \\
\text { JET PROPULSION LABORATORY } \\
\text { California Institute of Technology } \\
4800 \text { Oak Grove Drive } \\
\text { Pasadena, California } 91109\end{array}$} & 10. Work Unit No. \\
\hline & $\begin{array}{c}\text { 11. Contract or Grant No. } \\
\text { NAS7 }-918\end{array}$ \\
\hline & 13. Type of Report and Period Covered \\
\hline \multirow{2}{*}{$\begin{array}{l}\text { 12. Sponsoring Agency Name and Address } \\
\text { NATIONAL AERONAUTICS AND SPACE ADMINISTRATION } \\
\text { Washington, D.C. } 20546\end{array}$} & JPL External Publication \\
\hline & $\begin{array}{l}\text { 14. Sponsoring Agency Code } \\
\text { RE182 PX-644-11-00-0452 }\end{array}$ \\
\hline
\end{tabular}

15. Supplementary Notes

16. Abstract This publication presents a novel technique for simultaneously detecting data and estimating the parameters of a received carrier signal phase modulated by unknown data and experiencing very high Doppler, Doppler rate, etc. Such a situation arises, for example, in the case of Global Positioning Systems (GPS) where the signal parameters are directly related to the position, velocity and acceleration of the GPS receiver.

The proposed scheme is based upon first estimating the received signal local (data dependent) parameters over two consecutive bit periods, followed by the detection of a possible jump in these parameters. The presence of a detected jump signifies a data transition which is then removed from the received signal. This effectively demodulated signal is then processed to provide the estimates of global (data independent) parameters of the signal related to the position, velocity, etc. of the receiver.

One of the key features of the proposed algorithm is the introduction of two different schemes which can provide an improvement of up to $3 \mathrm{~dB}$ over the conventional implementation of Kalman filter as applied to phase and frequency estimation, under low to medium signal-to-noise ratio conditions. One scheme is based upon reprocessing (cycling) the measurements over an optimally selected interval while the altemative scheme proposes an adaptive Hilbert transform technique. The overall complexity of the proposed algorithm is about three times the camplexity of a single third order Kalman filter.

17. Key Words (Selected by Author(s))

Spacecraft Communications, Command, and Tracking; Communications; Navigation, Detection, and Countermeasures (General) Navigation and Guidance
18. Distribution Statement

Unclassified--Unlimited
19. Security Classif. (of this report) Unclassified
20. Security Classif. (of this page) Unclassified
21. No. of Pages

22. Price 